\begin{tabular}{|c|c|c|}
\hline Beitr. Ent. & Berlin & ISSN 0005-805X \\
\hline $\mathbf{4 9}(1999) 2$ & S. $489-530$ & 13.09 .1999 \\
\hline
\end{tabular}

\title{
JOSEPH SUGAR BALY: The man and his entomological works
}

\author{
Charles L. STaInes and Susan L. STAINES
}

\begin{abstract}
Summary
JOSEPH SUGAR BALY (1816-1890) was an English medical doctor who became a world authority on the Chrysomelidae (Coleoptera). From 1855 until 1891 he pablished 102 papers in which he described 217 genera, 2 subgenera, and 2.219 species. This article presents a brief biographical sketch, a bibliography of his entomological works, and a list of proposed taxa.
\end{abstract}

\section{Zusammenfassung}

Der englische Mediziner JoSEPH SUGAR BaLY (1816-1890) erlangte fitr die Chrysomelidae (Coleoptera) Weltgeltung. Von 1855 bis 1891 publizierte er 102 Arbeiten, in welchen er 217 Gattungen, 2 Untergattungen und 2.219 Arten beschrieb. Dieser Beitrag vermittelt eine kurze, biographische Ubersicht, eine Bibliographie seiner entomologischen Veröffentlichungen und eine Liste der vorgeschlagenen Taxa.

\section{Keywords}

Baly, biography; bibliography, proposed taxa.

\section{Acknowledgments}

We thank D. G. FURTH, Smithsonian Institution, and J. RUFFE, Royal Entomological Society, for sharing their biblographical resources on Baly. K. GRABOWSKI and P. FAWCETT (Field Museum of Natural History) were of great assistance in finding several Baly articles.

JOSEPH SUGAR BALY (1816-1890) was one of the most prolific chrysomelid wotkers. Except for some interest in the higher classification of Hymenoptera, all of BALY's work was on the Chrysomelidae. His work on many groups has not been superceded.

\section{Biographical Sketch}

BAlY was born in Warwick, England in 1816, and was the eldest son of Mr. JOSEPH BALY. He attended Grammer School under Rev. GEORGE INNES and was a student at St. George's Hospital, Hyde Park. BALY was also a pupil at the Shrewsbury Infirmary under Dr. BURD. After passing his examination he studied in Paris before setting up his medical practice in Leamington. BALY later purchased a large medical practice in London.

BALY's interest in entomology began when he was in his 30 's and was an outgrowth of his interest in microscopy. Prior to 1850 BALY purchased some insects from a dealer as objects to study with his excellent microscope. He became fascinated by insects and began devoting much of his leisure time to describing insects from around the world. 
BALY's entomological work was purely descriptive; he apparently had no interest in collecting insects. His interests were in Coleoptera and Hymenoptera, though he published no taxonomic works on the later group. BALY maintained a wide correspondence with field biologists such as BATES, CHAMPION, and WALLACE.

BALY's descriptions are remarkably detailed, relying on morphological characters rather than general color patten to distinguish species. He was one of the first entomologists to use male genitalia for separating species (1879). Unlike many entomologists of his time, BALY did not describe subspecies or color variations. When he did note different varieties they were noted as Var. A, etc.

Due to health problems related to overwork, BALY sold his London medical practice 1868 and moved to Warwick. He resumed a private medical practice, became the health officer for Leamington, a medical officer for the Union Infirmary, and was a magistrate for his borough. BALY was also appointed an honorary curator of the Warwick Museum. During this time BALY continued his entomological studies and was actively working on the genus Diabrotica when he died on 27 March 1890 after a brief illness. He was 73.

BALY married early and had one son and five daughters. BALY was described as quiet, amiable, and unemotional but having many friends.

BALY joined the Entomological Society of London (now the Royal Entomological Society) in 1850 , serving on the council from 1856 to 1859 . He was elected a fellow in the Linnean Society of London in 1865 . He was also a member of the Entomological Societies of France and Stettin.

BALY sold his Cassidinae to E. W. JANSON; part of his collection went to the British Museum between 1880 and 1905 , and part to a dealer. The British Museum material comprises over 28,000 specimens representing nearly 6000 species and includes 1551 types. BALY's Hymenoptera was purchased by the Hope Entomological Collections between 1857 and 1877 .

\section{Analysis of Baly's Proposed Taxa}

One measure of the value of a taxonomist's work is how proposed taxa have been accepted by other scientists. In 102 publications BaLY proposed 217 genus group names, 2 subgeneric group names, and 2219 species group names. Of the 217 generic names, 25 (20.7\%) are now synonyms and $8(3.7 \%$ ) are homonyms (SEENO \& WiLCOX 1982). The 53 names represent $24 \%$ of the names proposed.

Using the catalogs of the Alticinae (HEIKERTINGER \& CSIKI 1939, 1940), Galenucinae (WiLcoX 1971, 1972, 1973) and Hispinae (UHMANN 1957, 1958) Baly proposed 1347 species group names (354 Alticinae, 547 Galenucinae, 443 Hispinae). Of these $100(7.4 \%$ ) are synonyms, $26(1.9 \%)$ are homonyms, and $16(1.1 \%)$ are now subspecies. These 142 names represent $6.4 \%$ of the taxa proposed.

BALY's rate of unaccepted names is fairly high but not unusual for a worker who described species rather than did revisionary work. Many of the genera containing species group names have not been revised since BALY's death so the percentage of unaccepted names may change.

\section{Bibliography}

The author is J. S. BALY unless otherwise specified. The numbering system was devised for this publication. I have attempted to present BALY's articles in the order they were published. 
1. Description of two insects belonging to Pseudomela, a new genus of Chrysomelidae. - Transactions of the Entomological Society of London 4(1855): 87-88.

2. Monograph of the Australian species of Chrysomela, Phyllocharis, and allied genera. - Transactions of the Entomological Society of London (new series) 3(1855): 170-186, 241-263.

3. Descriptions of some new species of Chrysomelidae. - Transactions of the Entomological Society of London 4(1855): 339-352.

4. Catalogue of Hispidae in the collection of the British Museum. - London, $172 \mathrm{pp}$. (1858)

5. Description of Oxycephala imperialis $\mathfrak{n}$. sp. - Proceedings of the Entomological Society of London 1859: 88.

6. Descriptions of new species of phytophagous insects. - The Annals and Magazine of Natural History (3)3(1859): 195-209.

7. Descriptions of new genera and species of phytophagous insects. Fam. Galencinae. The Annals and Magazine of Natural History (3)4(1859): 55-61, 124-128, 270-275.

8. Descriptions of new species of phytophagous insects. - Transactions of the Entomological Society of London (2)S(1859): 146-161.

9. Descriptions of new genera and species of Eumolpidae. - Joumal of Entomology 1 (1860): 23-36.

10. Descriptions of some new species of Sagra; remarks on that genus; and the characters of Cheiloxerna, a new genus belonging to the same family. - Transactions of the Entomological Society of London (2)6(1860): 236-260.

11. Descriptions of six new species of Chrysomela from the east. - Journal of Entomology 1(1860): 93-98.

12. Descriptions of new genera and species of Phytophaga. - Journal of Entomology 1 (1861): 193-206, 275-302.

13. Descriptions of new species of phytophagous beetles. - The Annals and Magazine of Natural History (3)10(55)(1862): 17-29.

14. Descriptions of new genera and species of Phytophaga. - Journal of Entomology 1 (1862): 450-459.

15. Descriptions of new species of Cassididae, together with a list of all the species belonging to the same family collected by the late M. MOUHoT in Siam and Cambodia. - Journal of Entomology 1(1863): 6-14.

16. An attempt at a classification of the Eumolpidae. - Joumal of Entomology 2(1863): 143-163.

17. Descriptions of new Phytophaga. - Transactions of the Entomological Society of London (3)1(8)(1863): 611-624.

18. Descriptions of some uncharacterized genera of Phytophaga. - The Entomologist's Monthly Magazine 1(1864): 133-136.

19. Descriptions of new genera and species of Eumolpidae. - Journal of Entomology 2 (1864): 219-223.

20. Descriptions of genera and species of Hispidae. - The Annals and Magazine of Natural History (3)14(1864): 261-271, 334-345.

21. Descriptions of uncharacterized genera and species of Phytophaga. - Transactions of the Entomological Society of London (3)2(1864): 223-243. 
22. Further descriptions of new genera and species of Phytophaga. - Transactions of the Entomological Society of London (3)2(1864): 251-258.

23. Descriptions of the species belonging to the genus Paropsis, - Journal of Entomology 2(1864): 291-310.

24. Descriptions of new genera and species of Phytophaga. - Transactions of the Entomological Society of London (3)2(1864): 333-357.

25. Descriptions of new genera and species of Phytophaga. - The Annals and Magazine of Natural History (3)14(1864): 433-442.

26. Descriptions of new genera and species of Phytophaga. 8vo. Privately printed. - London. 16 pp. (1864)

27. Descriptions of new genera and species of Phytophaga. - The Annals and Magazine of Natural History (3)15(1865): 33-38.

28. Phytophaga Malayana: A revision of the phytophagous beetles of the Malay Archipelago. - Transactions of the Entomological Society of London (3)4(1)(1865-1868): $1-300$.

29. Descriptions of new genera and species of Phytophaga. - Transactions of the Entomological Society of London (3)2(1865): 427-440.

30. Descriptions of new species of Crioceridae. - The Annals and Magazine of Natural History (3)16(93)(1865): 153-160.

31. Descriptions of new genera and species of Gallerucidae. - The Entomologist's Monthly Magazine 2(1865): 97-101, 127-128, 147-148.

32. Descriptions of new species of Zeugophora, and a new species of Sphaeroderma. The Entomologist's Monthly Magazine 1(1865): 183-184.

33. in H. CLARK. An examination of the Halticidae of South America. - Journal of Entomology 2(1865): 375-412.

34. Descriptions of new genera and species of Gallerucidae. - The Annals and Magazine of Natural History (3)16(1865): 247-255.

35. Attempt at a classification of the Eumolpidae. - Journal of Entomology 2(1865): 433442.

36. Descriptions of new genera and species of Gallerucidae. - The Annals and Magazine of Natural History (3)16(1865): 402-410.

37. New genera and species of Gallencidae. - Transactions of the Entomological Society of London (3)3(1866): 471-478.

38. in H. ClARK. A catalogue of Phytophaga (Coleoptera, Pseudotetramera), Part 1. I. Sagridae, II. Donacidae, II. Crioceridae, IV. Megalopidae. With an appendix. Containing descriptions of new species by W. H. BATES and Rev. H. CLARK. - Williams and Norgate, London. $88 \mathrm{pp}$. (1866).

39. Characters of some undescribed species of Phytophaga belonging to the families Cassididae and Hispidae. - Transactions of the Entomological Society of London 1869: 83-90.

40. Descriptions of new genera and species of Hispidae; with notes on some previously described species. - Transactions of the Entomological Society of London 1869: 363382.

41. Descriptions of new genera and of some recently discovered species of Australian Phytophaga. - Transactions of the Entomological Society of London 1871: 381-400. 
42. Descriptions of some species of Cassididae new to science. - Transactions of the Entomological Society of London 1872: 59-72.

43. Occurrence of Vanessa antiopa in Great Britian and Ireland during the autumn of 1872. - The Entomologist 6(1872): 259.

44. Sphinx convolvuli, Tillus unifasciatus, and Ponera contracta near Warwick. - The Entomologist 6(1872): 259-260.

45. Catalogue of the phytophagous Coleoptera of Japan, with descriptions of the species new to science. - Transactions of the Royal Entomological Society of London 1873: 69-99.

46. Catalogue of the phytophagous Coleoptera of Japan, with descriptions of the species new to science. - Transactions of the Entomological Society of London 1874: 161217.

47. Honeybees. - The Entomologist 7(1874): 293.

48. Descriptions of new genera and species of Phytophaga. - Transactions of the Entomological Society of London 1875: 23-31.

49. Descriptions of hitherto uncharacterized species of Phytophaga. - The Entomologist's Monthly Magazine 12(1875): 73-75.

50. Descriptions of hitherto uncharacterized Australian Phytophaga. - Cistula Entomologica 2(1875): $45-50$.

51. Geănderte Namen. - Coleopterologische Hefte 14(1875): 213.

52. Descriptions of hitherto uncharacterized Phytophaga. - The Entomologist's Monthly Magazine 13(1876): 6-10.

53. Diagnoses of undescribed species of Phytophaga. - The Entomologist's Monthly Magazine 13(1876): 79-82.

54. Descriptions of hitherto uncharacterized Phytophaga. - The Entomologist's Monthly Magazine 13(1876): 126-129.

55. Descriptions of new species of phytophagous Coleoptera. - Cistula Entomologica 2(1876): 123-130.

56. Descriptions of new genera and species of Halticinae. - Transactions of the Entomological Society of London 1876: 433-449.

57. Descriptions of a new genus and of new species of Halticinae. - Transactions of the Entomological Society of London 1876: 581-602.

58. Descriptions of new genera and species of phytophagous beetles belonging to the family Cryptocephalidae, together with diagnoses and remarks on previously described genera. - Transactions of the Entomological Society of London 1877: 23-36.

59. Descriptions of new species of phytophagous beetles belonging to the family Eumolpidae; and a monograph of the genus Eumoplus. - Transactions of the Entomological Society of London 1877: 37-56.

60. Colias edusa at Warwick. - The Entomologists Monthly Magazine 14(1877): 89.

61. Diagnosis of a new genus and some undescribed species of African Phytophaga. The Entomologist's Monthly Magazine 14(1877): 177-182, 204-206.

62. Descriptions of new genera and of uncharacterized species of Halticinae. - Transactions of the Entomological Society of London 1877 ; 157-184, 283-323.

63. Descriptions of new species of phytophagous Coleoptera. - Cistula Entomologica 2 (1877): 179-185. 
64. Descriptions of new genera and species of Cryptocephalidae. - Transactions of the Entomological Society of London 1877: 211-232.

65. Descriptions of new genera and species of Galerucinae. - The Entomologist's Monthly Magazine 13(1877): 224-227.

66. Diagnoses of four species of Doryphora from Colombia. - The Entomologist's Monthly Magazine 13(1877): 273.

67. Descriptions of new species of phytophagous Coleoptera. - Journal of the Linnean Society of London, Zoology 14(1877): 336-356.

68. Characters of new genera and of some undescribed species of phytophagous beetles. The Annals and Magazine of Natural History (4)20(1877): 377-386.

69. Characters of new genera and of some new undescribed species of phytophagous beetles. - The Annals and Magazine of Natural History (5)1(1878): 38-44.

70. Descriptions of a new genus and of a new species of Halticinae. - The Annals and Magazine of Natural History (5)1(1878): 312-322.

71. Descriptions of some uncharacterized species of Crioceridae. - Cistula Entomologica 2(1878): 305-316.

72. Descriptions of three species of Doryphora from Peru and the Amazons. - The Annals and Magazine of Natural History (5)2(1878): 86-87.

73. Characters of undescribed species of Halticinae. - The Annals and Magazine of Natural History (5)2(1878): 223-233.

74. Description of new species and genera of Eumolpidae. - Journal of the Linnean Society, Zoology 14(1878): 246-265.

75. Descriptions of the phytophagous Coleoptera collected by the late Dr. F. STOLICZKA during Forsyth's Expedition to Kashgar in 1873-4. - Cistula Entomologica 2(1878): 69-383.

76. Descriptions of new genera and species of Gallerucinae. - The Annals and Magazine of Natural History (5)2(1878): 411-422.

77. Descriptions of genera and species of Australian phytophagous beetles. - Journal of the Linnean Society of London, Zoology 13(1878): 458-479.

78. Descriptions of new genera and species of South American Eumolpidae, chiefly from the Amazon region. - Transactions of the Entomological Society of London 1878: 281-298.

79. List of the phytophagous Coleoptera collected in Assam by A. W. CHENNELL. Esq., with notes and descriptions of the uncharacterized genera and species. - Cistula Entomologica 2(1879): 435-465.

80. Descriptions of new genera and species of Gallerucinae. - The Annals and Magazine of Natural History (5)3(1879): 73-84; 108-120.

81. An attempt to point out the differential characters of some closely-allied species of Chrysomela, principally those contained in Suffrian's 11 th group; also descriptions of some hitherto uncharacterized forms belonging to the same and other genera of the family. - Transactions of the Entomological Society of London 1879: 171-197.

82. Descriptions of phytophagous Coleoptera belonging to the families Chrysomelidae and Galerucidae, from Peru. - Transactions of the Entomological Society of London 1879: 235-259.

83. Descriptions of new species of Galerucidae. - Transactions of the Entomological Society of London 1881 : $51-59$. 
84. Descriptions of uncharacterized species of Eumolpidae, with notices of some previously described insects belonging to the same family. - Transactions of the Entomological Society of London 1881: 491-506.

85. The Colombian species of the genus Diabrotica, with descriptions of those hitherto uncharacterized. Parts I and II. - Journal of the Linnean Society of London, Zoology 19(1885): 213-229; 230-259.

86. Hispidiae. in F. D. GODMAN \& O. SALVIN (eds.). - Biologia Centrali-Americana, Zoology, Insecta, Coleoptera, Phytophaga. 6(2)(1885): 1-124.

87. Descriptions of new genera and species of Galentcidae. - Transactions of the Entomological Society of London 1886: $27-39$.

88. Descriptions of a new genus and of some new species of Galerucinae, also diagnostic notes on some of the older described species of Aulacophora. - Journal of the Linnean Society, Zoology 20(1886): 1-27.

89. Descriptions of uncharacterized species of Diabrotica. - Transactions of the Entomological Society of London 1886: 443-453.

90. Notes on Galerucinae and descriptions of two new species of Hispidae. - The Entomologists Monthly Magazine 23(1887): 268-270.

91. Description of a new species of phytophagous Coleoptera alleged to be destructive to the Dhan crops in the Chittagong. - Joumal of the Asiatic Society of Bengal (Calcutta) 55(1887): 412 .

92. Descriptions of three new Phytophaga from the east. - The Entomologist's Monthly Magazine 25(1888): 85-86.

93. Descriptions of some genera and species of Galerucinae. - Journal of the Linnean Society of London, Zoology 20(119)(1888): 156-188.

94. Viaggio di L. FEA in Birmania e regioni vicine. XIII. List of the Hispidae together with descriptions of some new species. - Annali del Museo Civico Naturale di Genova (2)6(1888): $653-666$.

95. Descriptions of new South American Coleoptera of the genus Diabrotica. - Proceedings of the Zoological Society of London 1889: 88-95.

96. Notes on Aulacophora and allied genera. - Transactions of the Entomological Society of London 1889: 297-309.

97. Diagnoses of uncharacterized species of Diabrotica. - The Entomologist's Monthly Magazine 25(10)(1889): 251-254.

98. Contributions à la faune Indo-Chinoise. Sagridae, Crioceridae, Chrysomelidae, Hispidae. - Annales de la Société Entomologique de France (6)9(1889): 485-492.

99. Descriptions of two new genera and of some uncharacterized species of Galerucinae. - The Entomologist Monthly Magazine 26(1890): 12-14.

100. On South American species of Diabrotica. Part I. - Transactions of the Entomological Society of London 1890: 1-86.

101. Scientific results of the second Yarkland Mission based upon the collections and notes of the late FERDINAND STOLICZKA PhD. Coleoptera Phytophaga. pp. 25-36. Office of Superintendent of Government Printing, India. Calcutta. (1890).

102. in C. J. GAHAN, On the South American species of Diabrotica. Part II. - Transactions of the Entomological Society of London 1891: $415-472$. 


\section{Obituaries and Articles on Baly, His Work, and His Collection}

ANONYMOUS. 1890. Obituary. - The Entomologist's Monthly Magazine 26: 142.

ANONYMOUS. 1890. Obituary. - Proceedings of the Linnean Society of London 1889-1890: 92-93.

ANONYMOUS. 1890. Obituary. - Zoologischer Anzeiger 13: 408.

ANONYMOUS. 1890. Obituary. Leopoldina 26: 111.

DARBY, $M$. in press. A biographical dictionary of British coleopterists.

F. 1892.Obituary. - Entomologisches Jahrbuch 1892: 195.

MUSGRAVE, A. 1932. Bibliography of Australian Entomology 1775-1930 with biographical notes on authors and collectors. - Royal Zoological Society of New South Wales. - $380 \mathrm{pp}$.

NEAVE, S. B. 1933. The history of the Entomological Society of London 1833-1933, - Richard Clay \& Sons. Bangay, Suffolk. - $224 \mathrm{pp}$.

NICHOLS, S. W. 1986. Early history of the use of genitalia in systematic studies of Coleoptera. - Quaestiones Entomologicae 22: 115-141.

SHARP, D. 1890. The late Joseph Sugar Baly. - The Entomologist 23: 197-200.

SMITH, A. Z. 1986. A history of the Hope Entomological Collections in the University Museum Oxford. - Clarendon Press. Oxford. - $172 \mathrm{pp}$.

SMITH, R. F. \& J. F, LAWRENCE. 1967. Clarification of the status of the type specimens of Diabroticites (Coleoptera, Chrysomelidae, Galenicinae), - University of California Publications in Entomology 43: 1-168.

WALSINGHAM, Lord. 1890. The President's Address. - Proceedings of the Entomological Society of London. 1890: Iviii.

\section{Taxa Proposed by BALY}

Alticinae

Acrocrypta n. gen. 14:457

aureipennis 53:435

dimidiata $14: 458$

mouhoti 14:458

Aedionychis

japonicus 46:189

Altica

amazona 62:163

facialis 82:239

Aphthona

amazona 62:299

chinensis $62: 295$

collaris $46: 198$

crassicornis $62: 295$

deyrollei $62: 296$

diversa 62:297

fulvipes 62:298

moerens $24: 343$

nigrocyanea $62: 298$ pilatei 62:296

pryeri $46: 198$

pygmaea 46:198 [hymonym, replaced by perminuta 52:213]

sordida $46: 197$

strigosa $46: 197$

verticalis $62: 297$

wallace $62: 178$

Apraea n. gen. 62:293

jansoni 62:294

Apteropoda

nigropicea $46: 207$

Argopistes

coccinelloides 46:202 [homonym, replaced by cocciniformis (CsIKI 1940)] Argopus clypeatus $46: 206$

fortunei 62:181

haroldi $56: 439$

orientalis $46: 206$ 
Arsipoda

caeruleata $62: 159$

erichsoni 73:232

femorata $25: 440$

fulvicollis $25: 440$

fulvipes $62: 284$

haematodera $62: 158$

lownei 25:441

macleayi 25:441

maerens 62:285

piceipes 77:477

rugulosa 25:442

wallacei $62 ; 285$

Asphaera

discofasciata 82:251

inclusa 83:57

limbifera $82: 252$

oblecta 83:56

Blepharida

chiliensis 29:432

flavopustulata $79: 441$

guttulata $83: 52$

nigripennis $83: 54$

ornata $83: 53$

xanthospilota $83: 54$

Camoena n. gen. 14:458

tibialis 14:459 [homonym, replaced by violaceipennis (JACOBY 1884)]

Cerichrestus

thammi 82:256

Chaetocnema

amazona $62: 306$

basalis $62: 310$

blanchardi 62:308 [replacement name

for Altica aenea BLANCHARD]

braziliensis $62: 307$

bretinghami $62: 170$

brevicornis $62: 317$

carinata $62: 174$

clypeata $62: 172$

cognata 62:168

concinnipennis $62: 170$

divergens 62:301

erichsoni $62: 175$

fuscomaculata 62:174

gravida $62: 302$ haroldi $62: 306$

labiata 62:305

laticeps $62: 315$

laticollis $62: 316$

madagascarensis 62:309

malayana $62: 312$

megalopoides $62: 173$

mexicana $62: 173$

natalensis $62: 166$

nitens $62: 312$

pallidicomis 62:303

parvula 62:310

persica $62: 167$

propinqua $62: 314$

robusta $62: 171$

rugiceps $62: 308$

sallei $62: 302$

separata 62:304

squarrosa $62: 169$

steinheili 62:304

submetallescens $62: 175$

wallacei $62: 171$

waterhousei $62: 315$

westwoodi $62: 311$

wilsoni $62: 313$

wollastoni $62: 167$

Charidea n. gen. 93:157 [homonym, re-

placed by Sangariola (JACOBSON 1922)]

fortunei $93: 158$

Chirodica

elongata $56: 442$

fulva $56: 442$

fulvipes 56:441

wollastoni $56: 442$

Clitea n. gen, 62:287

picta $62 ; 287$

Clyclophysa n. gen. 82:241

Crepidodera

africana $62: 159$

brasiliensis $24: 343$

collaris $62: 161$

costipennis $62: 161$

dimidiata $57: 586$

elegantula 24:342

flavescens 57:585

interrupta $57: 584$ 
japonica $62: 160$

madagassa 83:58 [homonym, replaced by balyi (DUVIVIER 1885)]

parallela 62:162

parvula $46: 195$

vestita $62: 162$

Cyclophysa n. gen. 82:241

albicornis 82:242

Diamphidia

bohemani 12:198

flexuosa $36: 403$

ornata 12:199

vittatipennis $36: 402$

Dibolia

duboulayi $62: 182$

gravida 57:599

intermedia 57:598

trimeni 57:599

Disonycha

erichsoni $82: 243$

fenestrata $73: 229$

ornata 53:80

pulchella $82: 242$

Docema

collaris 62:293

Elytropachys

caeruleipennis $56: 448$

quadripustulata 56:448

Enneamera

australis $53: 82$

fulviventris $53: 82$

limbata 53:82

thoracica $53: 82$

Epitrix

apicicornis $57: 589$

carinata $82: 238$

coeruleata $57: 592$

cyanella 57:593

nucea $57: 589$

segregata $57: 590$

sejuncta $57: 591$

serratula 57:591

subvestita $57: 588$

torrida 57:592

Eucycla n. gen. 56:439 [homonym, replaced by Schenklingia (CSIKI \& HEIKER-
TINGER 1940)]

aeneipennis $56: 440$

quadripustulata $56: 440$

Eugonia n. gen. 82:248 [homonym, repla-

ced by Eugoniola (CSIKI 1939)]

dimidiatipennis 82:249

Euphitrea n. gen. 48:27

assamensis 79:443

micans $48: 28$

wallacei $48: 28$

Euplectroscelis

bimaculata $62: 320$

deyrollei $62: 319$

nigripennis $62: 321$

placida $62: 320$

sordida $62: 321$

tibialis $62: 319$

Eutheca n. gen. 77:204

haroldi 77:205

malayana 83:55

Eutrea n, gen, 48:24

bowringii $48: 25$

Graptodera

angustata 46:191

caerulescens 46:190

flavicornis 46:192

fulvipennis $46: 193$

picipes 46:191

viridicyanea 46:191

Hermaeophaga

adamsii 46:193

nitidissima $82: 246$

tricolor 53:80

ventralis 53:80

Hyphasis

bevani 70:315

bipustulata 70:313

coccinelloides $70: 312$

indica 79:442

nigricornis 70:314

piceipennis $70: 313$

Iphitrea n. gen. 18:134

limbata 18:135

Lactica

apicicornis $53: 81$

binotata 53:81 
clypeata 82:244

fulvipes 53:81

jacobyi 82:244

nigripennis 53:81

peruviana 82:245

sellata 53:81

subnitida 53:81

Leptophysa n. gen, 62:165

batesii $62: 166$

Liprus

hirtus 46:194

Longitarsus

amazonus 62:177

buckleyi 62:291

capensis $62: 292$

concinnus $62: 290$

fryellus 62:291

peruvianus 82:249

scutellatus $62 ; 177$

wallacei $62: 292$

Lypnea n. gen. 56:446

flova $56: 446$

Medonia n. gen. 14:459

batesii $14: 459$

Megistops

ornatus $62: 322$

pretiosus $62: 322$

Monoplatus

fulvus 82:254

Myrcina

chapuisi $61: 205$

spectabilis $73: 232$

Nephrica

basalis $82: 240$

Niphraea n. gen. 69:40

hirtipennis $69: 40$

Nisotra n. gen. 25:437

bowringii 57:584

breweri $62: 157$

Nonarthra n. gen. 14:455

cyaneum 46:210

fulvum 46:211

ornata 14:456

variabilis $14: 456$

Notozona

batesii $29: 434$ clarkij 83:56

favipustulata 29:434

histrionica $29: 434$

jansoni 82:238

Octogonotes

limbatus 82:255

Oedionychis

batesii $7: 273$

bella 7:273

bifasciata 7:274

bilimbata 7:273

bitaeniata 73:223

chevrolatii $70: 322$

clarkii $70: 319$

crassa 73:226

elegans 73:224

germari $73: 228$

howittii $77: 478$

limbata 70:318

mouhoti 70:316

natalensis $73: 227$

nigrolineata $70: 321$

ornata $7: 274$

pretiosa 70:316

porosa 70:317

posticata 73:226

pulchra 82:253

quadrivitiata 7:274

recticollis $70: 319$

rugiceps $70: 320$

sallei 7:274

semifasciata 7:274

seriata $73: 225$

signifera $82: 252$

spilota 73:228

submarginata 7:274

tetraspilota 7:273

trivittata 7:274

virginella 7:274

Paradibolia n. gen. 48:31 indica 48:31

Pelonia

elegantula $82: 240$

Phrynocepha n. gen. 12:201

deyrollei $56: 444$

pulchella 12:201 
Phygasia

dorsata $73: 231$

hookeri $56: 445$

limbata 62:290

ornata 56:445

Phyllotreta

cumingii $62: 179$

downesi 62:300

funesta 46:196

jamaicaensis 62:299

malayana $62: 300$

orientalis $62: 178$

Physoma

violaceipennis 61:204

Platycepha n. gen. $77: 475$

eximia 77:476

Plectroscelis

australica 57:597

bella 57:595

compressipes 57:597

concinnicollis 46:208

cylindrica 46:208

discreta 57:596

granulifrons 57:596

granulosa 46:207

ingenua 57:594

philoxena 57:595

simplicifrons $57: 594$

Plectrotetra n. gen. 14:454

clarkii 14:455

Podagrica

chapuisii $56: 443$

madagassa 83:58

psyche 56:444

tarsata 56:443

Podontia

congregata $36: 405$

dalmani 36:405

flova 36:404

maculatissima 29:431

marmorata 36:403

mothoti 29:431

nigrotessellata $36: 404$

reticulata 36:404

rufocastanea $36: 405$

scaphoides 29:430 spectabilis $14: 452$

vittata $14: 453$

Prasona n. gen. 12:300

haroldi $73: 230$

varidis 12:301

Pseudodera n. gen. 12:200

orientalis $62: 286$

xanthospila 12:200

Psylliodes

angusticollis 46:209

bretinghami 14:457

chapuisii $62: 183$

difficilis $46: 210$

novaecaledoniae 57:600

punctifrons 46:209

quadridentata 57:601

Rhypetra n. gen. 48:30

costata $48: 30$

Sebaethe n. gen. 25:438

flavipennis 46:194

fulvipennis $62: 162$

nigricornis $62: 164$

pallidipennis 79:442

quadripustulata 56:80

torrida $62: 165$

Simaethea n. gen. $34: 247$ laportei $34: 248$

Sophraena n. gen. 24:342

ornata $24: 342$

Sphaeroderma

apicalis $46: 20$

apicipennis $62: 180$

fuscicornis 32:184

japana 46:204

ornata $62: 180$

picea $57: 582$

separata $46: 205$

seriata 46:203

tarsata 46:203

Sphaerometopa

cumingii $56: 434$

diversa $56: 434$

ornata 56:433

Sphaerophyma n. gen. $77: 478$

simoni $77: 479$ 
Sphaerophysa n. gen. 57:581 clavicornis 57:582

Stegnaspea n. gen. 62:181 trimeni $62: 182$

Stegnea n. gen. 82:247 nigripes $82 ; 248$

Stenophyma n. gen. 62:176 elegans $62: 176$

Sutrea n. gen. 56:435 albofasciata $56: 437$ bipustulata 56:438 elegans 56:436 hexaspilota $56: 436$ wallacei $56: 438$

Syphrea n. gen. 56:447 pretiosa $56: 447$

Systena batesii $25: 437$ caeruleata $62: 287$ deyrollei $62: 286$ oberithuri 73:229 ornata $62: 286$

Thyamis adamsii 46:199 amicula 46:201 bimaculata 46:200 binotata $57: 583$ godmani 57:583 inconspicua 46:201 [homonym, replaced by cervina $52: 213$ ] lewisii 46:199

Trichaltica amazona 57:586 elegantula 57:587 thammi 82:247

Xanthocycla n. gen. $48: 29$ chapuisii $48: 29$

Xenalica $\mathrm{n}$. gen. $48: 25$ murrayi $48: 26$ picea $48: 26$

Xenidea $\mathrm{n}$. gen. 14:453 alternata $14: 454$ purpureipei $62: 318$ wallacei $62: 318$

Xuthea n. gen. $34: 248$ orientalis $34: 249$

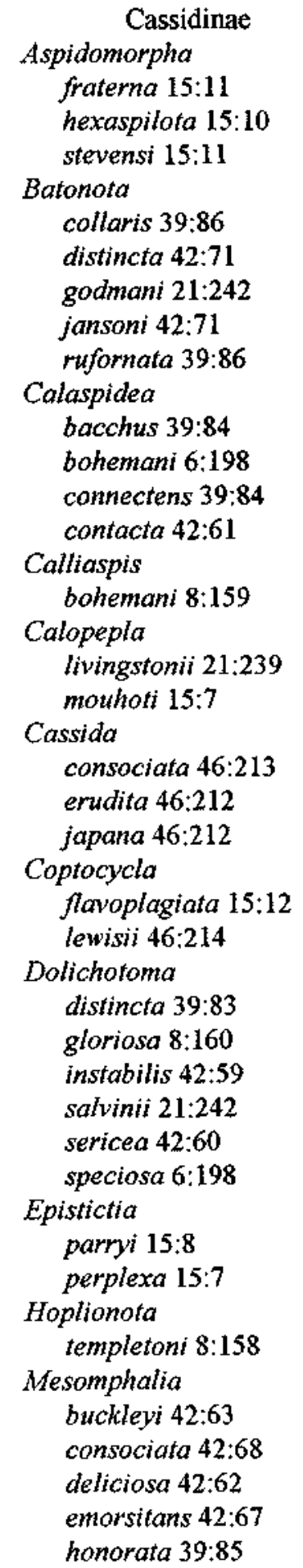


interjecta $42: 66$

latissima $42: 68$

pascoei 42:65

pauperula $42: 62$

pectinata $42: 64$

perjucunda 42:66

poecilaspoides $42: 69$

salvinii $21: 242$

Omaspides

abbreviata $42: 70$

bivittata 42:70

pulchella $6: 199$

Porphyraspis

pulchella 8:159

Prioptera

gibbosa 15:9

nigricornis 15:9

rugosa 15:8

Spilophora bohemani 6:197

deyrollei 15:6

speciosa 6:197

tetraspilota 6:196

Chlamisinae

\section{Chlaminus}

celebensis 28:59

chinensis 77:345

fulvipes $77: 345$

interjecta $45: 84$

lewisii $45: 83$

placida 77:344

spilota 45:85

velutina $77: 343$

wallacei $28: 58$

Diaspis

batesi $77: 342$

Exema

malayana 28:60

Chrysomelinae

Aesernia

magnifica 17:618

regalis $17: 618$

whitei 12:293

Augomela n. gen. 2:253
Australica

bartoni $2: 245$

cingulata $2: 245$

digglesii $27: 34$

erudita 13:24

geniculata $2: 248$

interruptofasciata 17:620

ioptera 2:246

pallida 2:248

pulchella 2:250

transversa 17:621

vittata $2: 250$

waterhousii 17:620

Augomela n. gen. 2:253 [made subgenus

of Australica in 8:156]

dives 8:157

elegans 2:256

iridea $2: 254$

ornata $8: 156$

pretiosa 2:256

pyroptera 2:254

Australica (Stethomela) n. subgen.

12:293

aeneipennis 12:293

gibbosa 13:25

parryi $21: 227$

scintillans 12:294

variabilis $17: 622$

Carystea n. gen. 27:33

inornata 27:33

jansoni $27: 33$

micans 53:80

Ceralces

ornata 12:198

spilota 21:227

walleri $21: 226$

Chalcolampra

chalybeata $2: 185$

decimpustata 17:619

marmorata $27: 35$

pustulata $2: 181$

simillima 2:185

thoracica 2:183

Chalcomela n. gen. $2: 258$

eximia $2: 260$

illudens 2:259 
insignis 2:259

intermedia 28:296

nigripennis 28:297

ornatissima 12:294

rubripustulata 28:297

sulcata 2:258

Chrysomela

adamsi 81:190

bonvouloirii 13:23

bowringii 11:96

cingulata 11:97

consimilis $46: 172$

eximia 13:20

fortunei $11: 94$

grutii 13:22

jacobyi 69:38

japana 46:171

krishnu 13:21

rufomarginata 81:191

separata 11:96

stalii 11:95

stevensi 13:23

templetoni 11:93

wallacei 13:21

Chrysomela (Atechna)

clarki 21:227

Colaphus

bowringii $27: 35$

Cryptostetha n. gen. 3:349

aenea 7:57

marmorata $3: 350$

rufipennis 7:58

suturalis 7:57

Cyclomela n. gen. $2: 257$

nitida $2: 257$

Cyclonoda n. gen. 77:474

Deuterocampta saundersi 77:356

Doryphora

amabilis 7:56

anchoralis $82: 235$

approximata $81: 194$

batesei $3: 343$

bella $3: 341$

bilunata $63: 183$

bivittaticollis $66: 273$ bohemanni 3:346 [homonym, replaced by stalii $7: 57$ ]

buckleyi 77:352

caerulea $7: 55$

cardinalis $7: 56$

chapuisi $72: 87$

congener 7:56

decipiens $82: 237$

degrandei 8:154

flexuosa 3:341

flavocincta 3:349 [homonym, replaced by fryella 7:57]

fulvopustulata 81:195

degrandei $8: 154$

godmani 81:194

haroldi $66: 273$

hebe $3: 344$

ingenua $66: 273$

irrorata 3:348 [homonym, replaced

by pluviata $7: 57]$

jacobyi 77:354

jansoni $63: 184$

joveti $3: 342$

jekelii 7:56

lurida 7:56

miniata $7: 57$

mirabilis 3:341

modesta $72: 86$

moesta 77:354

octodecimguttata 3:349

pulchella $66: 273$

salvinis 21:225

sheppardi $3: 343$

simulans $3: 347$

spectabilis $3: 341$

subglobosa $3: 346$

thammi 82:236

thomsoni 3:347

trivittata $3: 345$

verrucosa $77: 351$

undulata $77: 353$

vespertina $3: 345$

waterhousei $72: 87$

whitei $3: 348$

wollastoni $3: 344$ 
Dorysterna cruentata $3: 339$

festiva $3: 340$ pretiosa $3: 340$ tibialis $3: 339$

Elytrosphaera confusa $7: 58$ dejeanii 7:58 flovipennis 7:58 luridipennis 7:58 vittata 3:350

Entomoscelis assamensis 79:437 metallica 92:85

Eulina n. gen. $2: 170$

Eumaea n. gen. 27:37 pulchra $27: 38$

Eumela n. gen. 48:23

Gastrolina depressa 7:61 thoracica 21:228

Gonioctena aeneipennis 13:27 flexuosa 8:156 fortunet $21: 228$ murrayi 21:228 nigroplagata 13:28 rubripennis 13:28 scutellaris 13:27 thoracica 13:27

Horatopyga carinata 61:204 ornata 53:79 saundersi $53: 79$ sejuncta $53: 79$

Labidomera imperialis 77:355

Lamprolina $\mathrm{n}$. gen. 2:170 discoidalis 27:34 grandis 2:178 impressicollis 50:49 jansoni 50:48 perplexa 2:261 puncticollis $2: 179$ simillima $2: 178$
Leptinotarsa

kirschi 82:237

porosa 8:154

signatipennis $3: 352$

vittata $3: 351$

Lina

adamsii 21:229

aeneipennis 7:61

templetoni 7:60

Mesoplatys n. gen. 48:23

Micromela n. gen. 2:260

cupripennis 2:261

Nicea n. gen. $27: 36$

bella $27: 37$

dimidiatipennis 27:37

imperialis 27:37

Paralepta n. gen. 77:474 foveicollis $77: 475$

Paralina n. gen. 8:155

Paropsis

carnosa 23:307

chennelli 79:438

consimilis 23:306

fulvoguttata 23:298

geographica 23:303

ioptera 28:279

lownei 23:294

nigripicta 28:279

parryi 23:296

propinqua 23:306

roseola 23:308

sexmaculata $28: 280$

suspiciosa 23:297

tasmanica 23:294

wallacei $28: 280$

waterhousei $23: 296$

wilsoni 23:295

Phaedon

brassicae 46:174

incertum $46: 175$

Phratora abdominalis 75:375

Phyllocharis abdominalis 28:286

acroleuca 12:291 
apicalis 17:617

cyanipennis $2: 174$

eximia 69:39

flexuosa 2:175

jansoni 69:39

leoparda $2 ; 173$

melanospila 12:290

ornata 12:290

violaceipennis $12: 292$

viridiaenea $28: 286$

wallacei 12:292

Plagiodera

celestina 21:229

cinctipennis 13:26

cognata 27:36

cuprea 21:229

distincta $46: 174$

lownii 17:622

marginata 28:299

pallida 28:299

trimeni 13:25

viridivittata 13:26

walleri 27:36

Platymela n. gen. 2:241

sticticollis $2: 241$

unilineata $2: 242$

Polysticta

simsoni $61: 204$

Proseicela

chevrolatii $3: 351$

spectabilis 3:351

Pseudomela n. gen. 1:87

murrayi 1:88

natalensis 1:88

Sphaerolina n. gen. 41:400

Stethomela n. gen. 2:251

grandis 28:292

poroptera $2: 253$

prasina 2:252

quadripustulata 28:294

variabilis 28:293

Stenoplatys

pascoei 12:300

Stethomela

cornuta $50: 50$

fraternalis 50:49 limbata 50:50

Stilodes

cruciata 7:60

fenestrata 7:59

guttata 7:59

histrio 7:60

obsoleta 7:59

quadriguttata 7:60

scenica 7:60

Strumatophyma n. gen, 41:400

Aetheomerinae curtisii $28: 53$

oblita 28:54

pygidialis 28:55

Aspidolopha imperialis 28:50

Clythra

bella 28:48

distinguenda $28: 47$

japonica 45:79

Clythra (Diapromorpha) walleri 21:224

Chythra (Pantocometes) downesii 24:333

Clythra (Peploptera) tibialis 21:224

Coptocephala

dimidiatipennis 75:371

dubia $75: 370$

orientalis $45: 81$

Dinophthalma

consimilis $63: 180$

fasciata $63: 179$

nigriceps 63:179

Gynandrophthalma

lacordairii 28:52

malayana $28: 51$

ornatula $28: 53$

Megalostomis

coerulea $63: 183$

generosa 63:181

interruptofasciata 63:182

placida 77:341

Protophana

amazona 63:180

Themesia

grandis $63: 181$ 
Tibuboea

delectabilis $28: 45$

laportei $28: 44$

suspiciosa 28:46

\section{Criocerinae}

Crioceris

adonis 8:150

binotata $28: 29$

biplagiata 28:31

blackwellii 8:151

bohemani 17:612

clarkii 17:613

coronata $61: 177$

dromedarius 12:279

eximia 28:34

flavipennis 8:151

gibba 12:280

lateritia 17:613

obesa $17: 616$

obliterata $28: 30$

ornata $28: 28$

parvicollis 45:76

pfeifferi 17:614

pulchella $8: 15$

ruficollis $30: 155$

rugata 30:154

sallei 12:195

scabrosa $30: 153$

saundersi 28:35

scapularis 6:195

terminata 17:615

Lema

adamsii 30:156

amazona 71:311

ariadne 52:9

atriceps $28: 14$

atripennis $28: 26$

batesii 6:196

bimaculata 93:85

bipunctata $30: 157$

bisulcata 98:486

boisduvalii $28: 12$

bouchardi 71:307

bowringii 13:18

inghami 12:278 buckleyi 52:7

coeruleata 28:21

cognata 8:148

concinnipennis $30: 157$

connectens 28:13

constricta 28:18

coronata $45: 72$

degrandei 8:146

delauneyi 98:488

delicatula 45:75

dia $30: 158$

dilecta 45:74

discrepens 71:316

diversa 45:71

downesii 30:156

emarginata $52: 6$

erycina 8:147

ferox $28: 17$

fleutiauxi 98:487

fortunei 8:148

fraternalis 71:312

frontalis $13: 17$

globicollis $30: 158$

gravida 21:223

haroldi 52:7

hebe 8:150

honorata 45:73

idalia $30: 160$

jansoni $12: 277$

jekelii 12:279

kirbyi 71:305

latona $30: 160$

lewisii $45: 72$

lineatipennis 71:314

livingstoni 71:305

militaris 17:612

mouhoti 71:311

murrayi 71:308

mutabilis 28:11

mutabilis 71:308 [homonym, repla-

ced by danwini (CLAVAREAU 1904)]

monstrosa 28:16

nitidiceps 71:315

oculata $8: 149$ [homonym, replaced

by ecuadorica $38: 32]$

ornata $30: 159$ 
ornatula $71: 306$

parryi 12:277

pectoralis $28: 9$

perplexa 98:487

pithys 30:159

praeclara 30:159

psyche 30:157

pulchra 30:160

pulcherrima 71:307

quadrinotata 28:27

quadriplagiata 30:155

rufolimbata 52:9

salvini 71:313

saundersi $52: 8$

sellata $12: 278$

smithit 28:25

steinheili 71:312

stevensi 71:309

subapicalis 71:310

suturella 30:156

transversonotata 52:7

variolosa $8: 146$

vittatipennis $71: 315$

Stethopachys n. gen. 12:193

formosa 12:194

joveti 12:194

Acolastus

Cryptocephalinae

simsoni $64: 229$

Bucharis n. gen. 28:61

chapuisii 77:462

fulvipes 28:63

martius 77:463

suffriani 28:62

Cadmus

(Cyphodera) n. subgen. 58:25

chlamyoides 28:66

squamulosus 28:67

submetallescens 28:68

Chariderma n. gen. 58:28

pulchella 58:29

Coenobius

chinensis $64: 214$

discoidalis $64: 212$

fulvipes $64: 213$ lividipennis $64: 211$

picens 45:86

ruficollis $64: 212$

sulcicollis 45:86

Cryptocephalus

amatus $45: 96$ [homonym, replaced by

consalanus 46:217]

amazonus 64:221

amiculus 45:98

annulipes $28: 72$

apicipennis 28:71

approximatus $45: 93$

discrepans $28: 75$

discretus 45:97

fortunatus 45:94

gratus $64: 220$

histrionicus 64:221

instabilis 45:91

interjectus $75: 372$

jansoni 64:218

japanus $45: 92$

notatipennis $64: 219$

octospilotus $28: 73$

perelegans 45:88

permodestus $45: 95$

pilosus $45: 90$

scitulus 45:89

signaticeps 45:91

suspectus $28: 73$

tetradecaspilotus 45:89

wallacei $28: 74$

Dioryctus

grandis 28:64

lewisii $45: 87$

mouhoti 58:36

Ditropidus

amabilis 68:383

antennarius $68: 382$

biplagiatus $41: 389$

carbonarius $41: 284$

cornutus 68:384

costipennis 77:465

dimidiatus 41:38

duboulai 41:385

elegantulus 68:381

fascialis $77: 466$ 
fasciafus 41:390

fulvus 41:388

hirticollis 41:384

jacobyi 68:380

jansoni 77:466

laetus $77: 464$

laevigatus $68: 380$

odewahnii 41:387

ornatus 77:467

pascoei 68:381

phalacroides 77:464

pictus 68:382

pulchellus 77:468

rufocupreus 41:386

semicircularis 77:466

seremus 77:468

strigosus 41:385

subcylindricus $68: 385$

submetallescens 68:383

tarsatus 41:387

wallacei $68: 379$

Elaphodes

albohirstutus 41:283

Euphyma n. gen. 64:224

Idiocephala

bella $64: 225$

chapuisii $64: 224$

nigripennis $77: 459$

Lachnabothra

breweri 41:393

distincta 41:398

duboulai 41:399

integra 41:394

saundersii 41:397

waterhousei 41:396

wilsoni 41:395

Loxopleturus

laetus 28:77

Melixanthus

adamsi $64: 216$

bimaculicollis 28:65

coctus 28:65

placidus $64: 217$

Metallactus

eximius $64: 230$
Monachus

angulicollis $64: 215$

obscuricollis 64:215

Nyetra n. gen. 58:33

forcipata 58:34

Ochrosopsis

erudita 58:30

Pachybrachis contortus 64:231

eruditus 45:98

Paracadmus n. gen. 64:227 lucifugus 64:228

Paracephala n. gen. 64:222 pectoralis 64:223

Polyachus

bicolor 68:386

marginicollis 77:463

Prasonotus

morbillosus 58:35

ruficaudis 58:35

Rhombosternus

antennatus 77:460

gracillicornis 77:461

pretiosus $64: 226$

sulphuripennis 77:459

Stegnocephala n. gen. 58:32 discoidalis $64: 222$

Tappesia n. gen. 68:378 saundersi 68:379

Donaciinae

Donacia

aeraria $28: 7$

Adoxus

Eumolpinae

bowringii 9:27

nigripes 9:28

pollinarius $9: 28$

Aoria n. gen. 16:149

mouhoti 74:247

Apolepis n. gen. 16:161

aspera 16:161

Aulacolepis n. gen. 16:162

decorata 28:93

mouhoti 16:163 
Aulacia n. gen. $28: 268$

bipustulata 28:269

diversa 28:268

femorata 28:269

fulviceps 28:268

Aulexis n. gen. 16:153

nigricollis $16: 154$

varians $28: 82$

wallacei $28: 81$

Basilepta n. gen. 9:23

longipes $9: 23$

Bedelia

persica $74: 259$

Bromius

bohemani 19:220

cupreatus $28: 98$

evanescens 19:220

hebe 19:220

philippinensis 19:219

Callisina $\mathrm{n}$. gen. 9:30

fasciata $9: 30$

indica 84:503

mouhoti 26:11

quadripustulata 26:11

Callomorpha

imperalis 12:285

Chalcophana

binotata 84:501

eximia $84: 500$

jacobyi 84:501

mexicana 84:499

opulenta 84:499

Chalcophyma n. gen. 24:339

cretifera 24:339

laeta $24: 340$

tarsalis 24:340

tuberculosa 24:341

Chalcoplacis n. gen. 24:338

alternata 78:286

cupreata $78: 288$

echinata 78:287

elephas 78:284

femorata $78: 283$

hirticollis $78: 284$

ingenus 78:285 nitidicollis $78: 286$

sumptuosa $24: 338$

Cheiloxena n. gen. 10:254

westwoodi 10:255

Cheiridea n. gen. 74:253

chapuisi 74:253

Chrysochus

chinensis 7:125

mouhoti 19:222

pulcher 26:1

thoracicus 7:125

Chrysodina n. gen. 19:221

igneicollis 19:221

nigrita $78: 282$

Chrysolampra n. gen. 7:126

mouhoti 19:220

piceipes 79:440

splendens 7:126

Chrysopida n. gen. 12:288

adonis 12:289

festiva 12:289

insignis 28:161

murina $28: 162$

regalis $26: 11$

Clisithera n. gen. 19:220

nigricomis 19:221

Colaspis

dimidiata 9:35

elegantula $24: 341$

humeralis $9: 35$

lefevrei 59:37

Colaspoides

biplagiata $28: 137$

coerulescens 28:145

coerulipes 28:144

cognata 28:149

cuprea 28:138

deyrollei 78:294

dorsata $78: 293$

elegans $28: 143$

fuscoaenea 28:140

inornata 28:137

insignis 28:142

laportii 28:148

micans 28:140 
modesta 28:136

ornata 78:294

parvula 28:152

philippinensis 28:148

picea $28: 151$

puncticeps 28:141

quadripartita 28:152

rafflesii $28: 147$

regularis $28: 136$

robusta 28:139

simillima 28:139

tuberculata 28:150

varians 28:135

violacea $28: 151$

viridana 28:146

viridimarginata $28: 142$

Colasposoma

abdominale 26:15

auerovittatum 26:14

coeruleatum $79: 440$

cumingii 28:271

distinctum 28:272

downesii 13:19

fulvicorne 29:428

gratiosum 26:15

igneicolle 29:428

inconstans $26: 14$

mutabile 28:273

nigriventre 28:273

nitidum 28:272

pretiosa 9:36

propinquum 28:274

pulcherrimum 26:15

rugulosum 28:275

sellatum 74:254

tibiale 61:178

varians $61: 178$

viridiaeneum 29:429

viridivittatum 29:430

Corycia n. gen. 19:221 [homonyn, repla-

ced by Corysthea 30:336]

funesta 19:222

Corynodes

assamensis 79:439

aureipennis $28: 102$

caeruleatus $28: 122$ cupreatus 28:107

decminotatus 9:31

gloriosus $7: 125$

hopei $26: 7$

igneofasciatus $9: 32$

igneipennis 28:122

limbatus 84:505

lorquinit 28:118

propinquus 28:113

pulchellus 9:31

pyrospilotus $9: 32$

trilobatus 28:129

viridanus 28:105

Corysthea [see note on Corycia]

ferox 24:336

Cychrea n. gen. 19:222

histrio 19:222

Dematochroma n. gen. 26:16

picea $26: 16$

Demotina n. gen. 16:158

bivittata $28: 86$

bowringii $16: 159$

decorata 46:163

fasciata $46: 162$

fasciculata $46: 162$

grisea $28: 87$

jansoni $28: 89$

modesta $46: 164$

murina 28:86

ornata $28: 90$

parvula $28: 89$

pauperata $28: 88$

rufopicea $28: 90$

rugicollis $28: 85$

serraticollis $28: 85$

scutellata 16:159

wallacei $28: 87$

Dermorhytis n. gen. 12:282 [homonym, replaced by Abirus (CHAPUS 1874)]

apicalis 28:264

elegans $26: 8$

fortunei $12: 283$

igneofasciata $12: 283$

ornatissima $26: 8$

philippinensis $28: 263$

piceipes 28:265 
Dermoxanthus n. gen. 7:126 fraternus 7:127 fulvus 7:126

Dictyneis n. gen. $35: 434$ spilotus 24:341

Endoxus n. gen. 12:285

Epiphyma n. gen. 9:29

Eriphyle n. gen. 19:222 unimaculata 19:222

Eryxia n. gen. 35:437 baikii $35: 437$

Eubrachis n. gen. 74:248 indica $74: 249$ spinipes $74: 248$

Eulampra n. gen. 78:281 batesi $78: 282$

Eumolpus australis 59:50 batesii 59:53 carinatus 59:54 imperialis 59:51 nitidus 59:48 separatus $59: 47$ speciosus 59:52

Eurydemus jansoni $74: 258$

Euryope cingulatus $9: 33$ monstrosa 13:19 nigrita 84:505 pulchella 84:504 terminalis $9: 34$

Geloptera n. gen. 12:283 eximia 28:266 geniculata 12:284 igneonitens 77:472 purpurata $28: 267$ tuberculata 12:284 vestita $77: 473$

Glyptoscelis aeneipennis $24: 334$ albicans $24: 334$ fascicularis 24:334

Hemiplatys n. gen. 16:160 pascoei $16: 160$ Iphimeis n. gen. 18:133 fulvipes 18:134

Jansonius n. gen. $74: 264$ alternatus 74:264

Lamprosphaerus n. gen. 7:124 abdominalis $7: 124$ aeruginosis $7: 125$ biplagiatus $78: 290$ collaris $7: 124$ diversicornis 78:289 fulvitarsis 78:289 generosus 78:291 hebe $24: 337$ lateralis $24: 338$ lateralis $78: 292$ [homonym, replaced by balyi (LEFÈVRE 1885)] pulcher 78:291 quinquepustulatus 24:337 ruficeps 78:292 scintillaris $24: 338$ specularis 7:125 tarsatus 7:124

Lepina n. gen, 16:161 inconspicua $16: 162$

Lepronida n. gen. 19:221 batesii 19:221

Leprotes n. gen. 16:158 fulva $74: 250$ lewisi $74: 251$

Lophea n. gen. 35:441 melancholica $35: 442$

Lypesthes n. gen. 16:152

Macrolema n. gen. 12:275 vittata $12: 275$

Menius concinnicollis $61: 179$ costatus $61: 178$ murrayi $61: 178$

Meroda n. gen. 9:29 costata $9: 29$ fulva 12:197 rufipennis $12: 197$

Metaxis n. gen. 16:157 [homonym, replaced by Hyperaxis (GEMMINGER \& HAROLD 1874)] sellata $16: 157$ 
Metaxyonycha

batesi $84: 495$

distincta $84: 497$

gigas $84: 491$

octosignata $84: 496$

pretiosa $84: 494$

pulchella $84: 493$

retifera $84: 498$

tarsata 84:497

Mouhotia n. gen. 74:262 [homonym, re-

placed by Mouhotina (LEFEVRE 1885)] femorata 74:262

Myochrous

armatus 24:335

expanatus $24: 335$

sallei $24: 335$

Neculla n. gen. 16:152

Nephrella n. gen. 16:155

elongata $16: 155$

Nodina

ceramensis $28: 262$

chalcosoma 46:170

fulvipes 28:260

gigas $28: 260$

minuta 28:261

separata 28:261

Nodostoma

aeneipenne 28:235 [homonym, repla-

ced by haroldi (JACOBY 1908)]

aeneomicans 28:237

affine $28: 243$

amboinense $28: 250$

anthracinum 28:247

apicale 28:213

armatum 28:226

aureacupreum 28:232

basale 28:238

bevani $59: 40$

bipustulatum $28: 227$

bohemani $26: 14$

bohemani 28:220 [homonym and

synonym (KIMOTO 1985)]

castaneum 28:246

collare 28:234

concinnicolle $75: 373$

consimile $46: 168$ costata $26: 12$

cumingii 26:14

cupreatum 28:231

cupripenne 28:231

cylindrica $26: 13$

diversipes $28: 253$

dormeri $59: 39$

elegantulum 28:216

evanescens 26:13

flowopustulatum 46:169

foveicollis $26: 12$

frontale $28: 253$

fulvipes 28:228 [homonym, replaced by xanthopus (HAROLD 1874)]

gratum 28:224

hirticolle 46:167

humerale 28:219

imperiale 28:246

jansoni 26:13

javanense $28: 224$

laeve $28: 220$

laterale 28:218

lateripunctotum 28:230

magnificum 59:38

nigricornis $26: 13$

nigripes $26: 12$

nigritum 28:248

nigroaeneum 28:239

nigrum 28:248

nitidum 28:237

pallida $26: 13$

pallidipes 28:250

pallidulum 46:169

piceipes 28:241

piceomaculatum 28:222

piceum 28:227

pictum 28:225

placidum 28:221

plagiosum 75:373

proximum 28:214

pulchellum 28:242

purpureipenne 28:249

scabrosa $26: 12$

semivittata 26:13

strigicolle 28:240

tibiale 28:245 
tricolor 59:38

trivittatum 28:213

tuberculatum 28:229

viride 28:236

viridiaenea $26: 12$

viridiornatum 28;251

wallacei 28:216

Ocnus

pallidus 74:255

Pachnephorus

bretinghami 74:256

clypeatus 28:94

convexicollis 28:95

lewisi 74:257

porosa $74: 257$

torridus $74: 256$

viticollis $28: 95$

Parascela n. gen. 74:252

Paria

cuprescens 75:374

robusta $46: 166$

variabilis $46: 166$

vittaticollis 78:297

Platycorynus

aeneus 26:5

approximatus 26:6

biseriatus $26: 4$

coelestinus 26:6

congener 26:3

costatus $26: 2$

cumingii $26: 3$

cupreus $26: 6$

dohrnii 26:4

elegantulus $26: 5$

fabricii $26: 4$

fraternus 26:5

fuscoaeneus 26:6

gratiosus $26: 7$

ignitus 26:6

longicornis $26: 2$

marshalli 26:2

mutabilis $26: 3$

mouhoti 26:7

parryi 19:223

parvulus 26:7

perplexus 26:5 pretiosus $26: 4$

robustus 26:3

sheppardi $26: 7$

stevensi 26:5

tuberculatus 26:3

waterhousii $26: 4$

Piomera n. gen, 16:156

brachialis 16:157

Pseudocolaspis

eximia 59:44

femorata $74: 261$

lefevrei $74: 260$

longicollis 74:259

murrayi 12:197

rigida $59: 43$

Pyropida n. gen. 14:450

biplagiata $26: 15$

elegantula $26: 15$

lateralis $26: 15$

nigrocaerulea 26:16

sumptuosa 14:451

Rhyparida n. gen. 12:286

alternata $26: 9$

amabilis 28:193

amboinensis 28:204

angulicollis 28:211

approximata 28:184

aruensis $28: 186$

basalis 28:168

bipustulata 28:199

brunnea 28:196

celebensis 28:210

confusa 28:187

cupreata 28:177

dimidiata $12: 286$

dimidiatipennis $26: 8$

distincta 28:192

divresa 28:192

dorsata $26: 8$

elevata 28:205

fasciata $26: 10$

femorata $26: 10$

formosa $59: 40$

fraternalis $28: 174$

frontalis $28: 181$

fulva 26:11 
fulvescens 28:194

fulviceps 26:10

fulvipes 28:189

geniculata 12:288

grandis 12:287

horsfieldii $28: 202$

howitii 59:41

impressicollis 28:176

impuncticollis 26:11

inconspicua 28:194

inconstans 26:10

intermedia 28:188

instablilis 28:187

javanensis 28:196

labiata 28:171

lateralis 28:210

laterivittata 28:207

laticollis 28:178

limbata 26:9

lorquinii 28:166

maculicollis 77:473

moesta 28:178

nigripennis 26:10

nigroaenea $26: 9$

nucea 28:204

obliterata 28:197

obsoleta 28:185

opacicollis $28: 195$

ovalis 28:209

parvula 28:208

pascoei 26:10

picea 28:179

picta 28:200

pinguis 28:209

placida 28:190

pulchella 12:287

puncticollis 28:169

purpurea 28:174

regularis $26: 9$

scutellata 28:175

semipunctata $28: 169$

separata 28:191

sordida $26: 10$

submetallica 28:176

sulcata 26:8

sulcicollis 28:199 suspecta 28:190

tibialis 28:183

trilineata $26: 9$

tristis 26:8

tumifrons 28:198

variabilis 28:182

vittipennis $26: 9$

wallacei 28:200

Scelodonta

albidovittata 59:42

bidentata 59:43

granulosa 28:158

jacobyi 61:177

lewisii 46:165

murrayi 29:427

natalensis $61: 177$

nitidula 26:2

pulchella 26:1

purpureomaculata 26:1

simoni 74:251

Spilopyra n. gen. 9:24

sumptuosa 9:25

Stasimus n. gen. 16:150 rugosus $16: 150$

Stenolampra $\mathrm{n}$. gen. 7:127

costata 7:127

geniculata 7:127

Stethotes n. gen. 28:254

apicicornis 28:256

atra 28:259

consimilis 28:257

longicollis $28: 257$

nigritula 28:258

tarsata 28:258

Syagrus

rugifrons $74: 263$

Syricta n. gen. 35:440

Terillus

duroulayi $77: 471$

foveolatus 77:469

perplexus 77:470

squamosus $77: 470$

vittatus $77: 471$

Trichotheca n. gen. 9:26

hirta 9:26 
Trichochalcea n. gen. 78:295

aeneipennis 78:296

rugata 78:296

Trichochrysea n. gen. 12:195

mouhoti 12:196

vestita 12:195

Typophorus

basalis 7:128

humeralis $7: 128$

kirbyi 7:128

obliquus 7:128

quadripustulatus 7:128

ruficollis 7:128

Xanthonia n. gen. 16:151

placida 46:161

stevensi 16:15l

Xanthopachys n. gen. 26:16

nigripes $26: 16$

\section{Galerucinae}

Acrocrypta

coccinelloides $65: 224$

pallida 65:224

purpurea 65:224

Acroxena n. gen. 79:462

nasuta 79:463

Adorium

bowringii 17:623

circumdatum 12:296 [homonym, re-

placed by limbipennis (WEISE 1924)]

collaris 12:295

japonicum 46:176

ornatum 12:295

sordidum 29:435

tarsatum 29:435

Aenidea n. gen. 46:179

abdominalis $46: 180$

armata 46:179

barbata 79:464

basalis $46: 181$

eximia 79:464

facialis $87: 27$

nigripes $46: 182$

ornata $46: 180$

Aethonea n. gen. 31:100

murrayi 31:101
Agelastica

caerulea $46: 188$

orientalis $75: 379$

Agetocera

hopii 29:438

humeralis $80: 108$

lobicornis 29:437

melanocephala 80:109

Alopena n. gen. 21:239

collaris 21:239

Antipha n. gen. 34:251

bretinghami 80:118

chinensis 80:115

costata 80:116

discoidalis 80:117

flavofasciata 79:456

frontalis 80:117

histrio 79:456

nietneri 80:116

picipes $34: 251$

posticata 79:455

pretiosa 80:117

pulchella 80:116

Aplosonyx

elongato 17:624

Arcastes n. gen. 31:147

biplagiata 31:147

Arthrotus

cyaneus 46:184

variabilis $46: 183$

Astena n. gen. 31:127

atripes $31: 128$

Atysa n. gen, 21:238

fulvicornis 87:33

funesta 86:33

jansoni 86:32

nitidicollis $86: 32$

sulcicollis 86:32

terminalis 21:239

Aulacophora albofasciata $87: 6$

antennata 87:21

apicicomis 96:306

approximata 87:12

armigera 96:305

bipartita 93:183 
biplagiata $96: 307$

boisduvali $87: 10$

cornuta 79:445

costipennis 87:26

diversa 96:306

downesi 87:20

duboulayi $87: 22$

excovata 87:18

excisa 87:25

fabricii $87: 14$

flaviventris $87: 13$

frontalis 93:181

instabilis 87:10

laevifrons $93: 185$

lata 87:8

lewisit $87: 24$

mouhoti $87: 25$

nigroscutata $87: 14$

nigrosignata $87: 23$

occipitalis 93:184

octomaculata 87:17

olivieri $93: 184$

perplexa $79: 447$

perroudi 93:177

propinqua $87: 11$

pulchella 79:446

pygidialis 87:7

quadriplagiata 46:186

scutellata 61:205

semilimbata 87:24

stevensi $87: 22$

tetraspilota $87: 17$

wallacei $87: 15$

wilsoni 93:177

Berecyntha n. gen. 31:98

tibialis 31:98

Boneisa n. gen. $31: 100$ clarkii 31:100

Buphonida n. gen. 29:437

evanida 29:437

placida $86: 38$

puncticollis $86: 38$

submarginata $86: 38$

Byblitera n. gen. 18:136

deyrollei 18:136
Caritheca n. gen. 65:226 quadripustulata $65: 226$

Cerochroa maculicollis 21:232

Cerophysa

flava 86:28

wallacej $65: 227$

Cerotoma

altemata $37: 477$

amazona 37:475

congener $37: 473$

contubernalis $37: 475$

deyrollei $37: 477$ [transferred to Dia-

brotica and replaced by stevensi

87:248]

degrandei $37: 472$

erichsoni $37: 478$

excavata $37: 474$

heterocera $37: 472$

limbifera $37: 475$

perplexa 37:476

pulchra 37:476

quadripustulata $37: 477$

salvinii $37: 478$

transversofasciata $37: 474$

Charaea n. gen. 75:376

flaviventre 75:376

Chorina n. gen. $37: 471$

Chthoneis n. gen. 18:135

albicollis 76:421

apicicornis 18:136

bivittata $76: 421$

grayi $76: 422$

Clitena n. gen. 21:229

igneipennis 31:99

limbata 21:230

melancholica 21:231

Cneorane n, gen. 31:97

elegans 46: 182

fulvicollis 31:97

Cneorats n. gen. 86:27

rugulipennis 86:27

Coelomera

batesii $29: 439$

bipusiulata $24: 344$ 
cinixa 29:440

laeta $24: 344$

ornata $29: 440$

ruficornis $24: 343$

Cymorta n. gen. 34:249

apicipennis $93: 162$

facialis 93:162

ocellata $93: 161$

porrecta $34: 250$

Diabrotica

abbreviata $85: 238$

adornata 100:17

adonis $7: 272$

aegrota $95: 90$

aeneipennis $85: 234$

aeneiventris 95:95

albidocincta 102:431

albocincta 95:93

albomarginata $95: 92$

albosignata $89: 453$

albovittata 95:95

alcyone 95:94

alternata 85:244

amabilis $85: 222$

amplicornis 89:446

analis $100: 52$

apicalis 85:244

apicipennis 100:52

arcuata 7:271

asignata 100:78

atriceps 100:76

atrilineata 97:252

atrilineata 100:58 [homonym, repla-

ced by deliqua (WEISE 1921)]

atriscutata 100:77

atritarsis 100:77

atromaculata 95:90

atrosignata 100:46

bartleti 100:8I

batesii $7: 271$

beata $85: 244$

bella $85: 247$

bilineata 100:20

bipartita $95: 95$ [homonym, replaced

by denotata (GAHAN 1891)]

bipustulata $85: 221$ bisecta 100:14

bivittaticollis $85: 257$

borrei 97:253

brevicornis 100:51

brevivittata 100:55

buckleyi 80:80

buqueti $97: 252$

butleri $85: 251$

caviceps 97:253

cavicollis 100:55

chapuisi $85: 227$

chloris 100:84

cinctipennis 89:449

clarkella 7:271

clarkella 95:89 [homonym, replaced

by clarki (WEISE 1916)]

clypeato 85:249

coccinca $24: 345$

cognata 95:93

confluenata 100:26

confraterna 95:90

consentanea 85:214

consimilis $89: 447$

contigua $95: 88$

cornuta 89:448

corrugata 97:254

coryphaea $85: 233$

crenulata 100:83

cruciata 97:253 [homonym, replaced

by crucigera (WEISE 1916)]

curtisii 89:449

curvipustulata 100:44

decaspila 100:16

deliciosa 85:251

deyrollei $24: 347$

dilaticornis $80: 81$

dimidiatipennis $24: 350$

discoidalis $24: 349$

discrepans 95:94

divisa $80: 83$

diversicolor 100:86

diversicornis 100:63

donckieri 97:252

duodecimsignata 95:90

duvivieri $89: 445$

dysoni $88: 217$ 


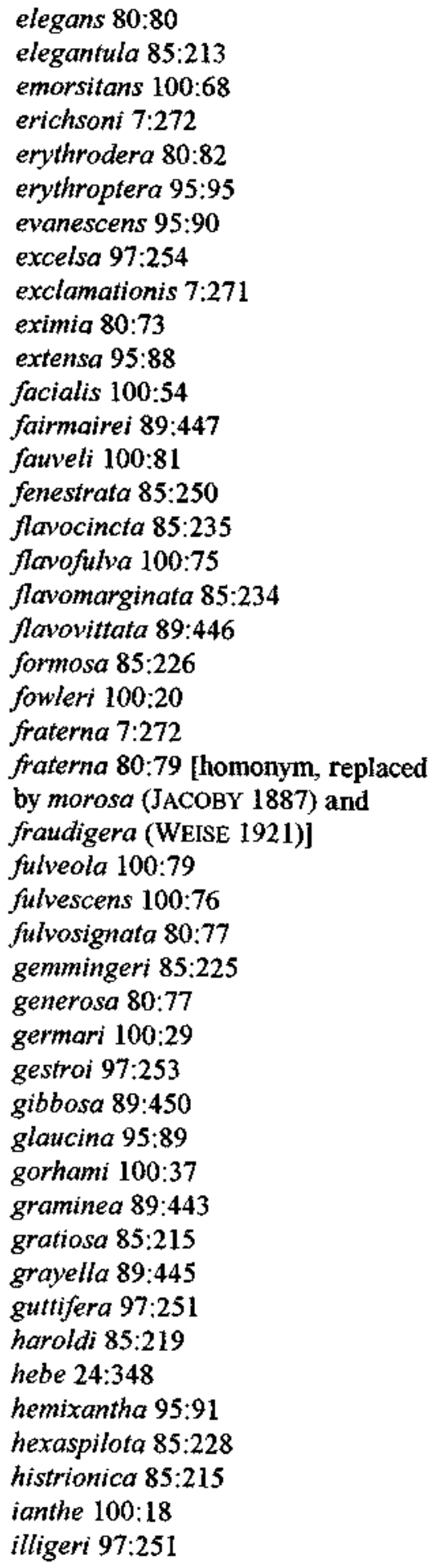

inaequalis $85: 219$

incerfa 85:236

inconstans 85:224

insignita $85: 256$

intermedia 85:252

interrupta 89:445

interruptofasciata $80: 75$

interruptolineata 95:91

jacobyi 80:76

javeti $97: 251$

jekelii 85:246

jucunda 85:247

kirbyi 100:57

kirschi 85:231

klugii 85:258

kraatzi 100:35

labiata $85: 223$

laetabilis $85: 257$

lata 89:451

lativittata $85: 244$

lebasii 85:221

leucospila $85: 253$

limbata 95:91

limbella 85:218

limbatipennis 95:93

limbifera 82:257

lugubris 85:253

lutescens 100:77

luteopustulata 100:43

maculatipennis 102:436

mediofasciata 100:14

mediovittata 85:237

melancholica 95:92

melanocephala 89:455

melanopyga 95:88

meyeri 100:63

mutabilis $85: 225$

nigriceps $80: 75$

nigritarsis 98:252

nigrocincta $89: 451$

nigroguttata $85: 243$

nigrolimbata 85:218

nigronotata 85:216

nigroscutata 100:65

nigrostriata $100: 69$

nigrovitulata $85: 242$ 
nitidicollis $95: 88$ notaticollis $95: 91$ oculata 100:72 octopustulata 100:19 octosignata $80: 76$ oberthuri $89: 452$ ornata $7 ; 273$ ornatula 85;224 opacipennis $80: 82$ pallescens 100:80 paraensis $89: 452$ pascoei $80: 78$ pauperata 100:27 pectinicornis $97: 252$ perplexa $85: 237$ perspicua 95:94 piceicollis 89:453

picicornis 95:89 piceolimbata 100:73 piceolineata 85:243 piceomarginata 100:72 picopicta 100:25 picosignata 100:61 placida 85:220 posticata 85:252 prasinomarginata 100:71 propinqua 85:255 proximans 100:51 puella 85:238 pulchra 24:345 puncticollis $24: 346$ quadrivittata 24:349 [homonym, replaced by spilothorax (HAROLD 1875)] reedi $100: 33$

regalis $7: 270$

reitteri 100;66

reticulata 97:254

robusta $85: 255$

rufolimbata $80: 74$

rugata 80:84

rugulipennis 100:19

rugulosa 89:450

rugulosa 100:64 [homonym, replaced by subrugosa (GAHAN 1891)] sallei $85: 227$ sanguinipennis 102:466

saundersi $24: 350$

schaufussi 100:74

scutellata $95: 89$

sedata 100:48

separata $85: 232$

serraticornis 85:255

setifera 82:257

setosa $89: 454$

sexmaculata $80: 81$

simulans $85: 222$

simulans 95:93 [homonym, replaced

by amazona (WEISE 1916)]

similata 85:235

simulata 100:50

sordidipennis 97:253

soroensas 100:54

speciosissima $80: 79$

spectabilis 102:443

spectanda $85: 241$

spilota 85:216

spiloptera $85: 242$

spiloptera 95:92 [homonym, replaced by melanospila (GAHAN 1891)]

stali 95:94

staudingeri $97: 254$

steinheili $85: 240$

stevensi 85:248 [replacement name

for Cerotoma deyrolli 37:447]

suoveola 95:94

sublimbata $24: 347$

submarginata 100:67

subsimilis 102:456

subsulcata $24: 351$

suffusa $85: 229$

stuturalis 24:348 [homonym, replaced by tropica (WEISE 1916)]

tarsalis $97: 254$

tenella 24:348

testaceicollis 100:9

tetraspilota $24: 351$

tetraspilota 85:254 [homonym, replaced by socia (GAHAN 1891)]

theimei 85:231

tibialis 100:79

torta 97:254 
transversa 100:34

trifoveolata $100: 58$

triplagiata $7: 272$

tuberculata 95:91

unifasciata $95: 92$

vagrans $95: 90$

vespertina $7: 271$

virescens $85: 223$

virginella $85: 228$

viridana $89: 443$

viridans $95: 89$

viridilimbata $80: 74$

viridipustulato $85: 226$

vittatipennis $89: 454$

volxemi $97: 253$

weise 100:53

westwoodi $97: 252$

xanthoptera $85: 250$

zonula 95:97

Dircema

columbicum $36: 408$

discoidale 36:406

laetum 36:408

laticolle 36:406

modestum 36:409

pulchrum 36:407

sordidum 36:409

Doridea n. gen. 21:236

insignis 21:236

Doryida n. gen. 31:97

mouhoti $31: 98$

nigripennis $99: 12$

tarsalis $99: 12$

Doryxena n. gen. 12:202

Emathea n. gen. 31:147

aenipennis $31: 148$

geniculata $79: 451$

violaceopennis $99: 12$

Eumaea n. gen. 27:37 [homonym, repla-

ced by Galerumaea (HINCKs 1949)]

albofasciata $86: 36$

fasciata $86: 36$

flavipennis 86:37

Euphyma n. gen. 79:457

collaris 79:457

Eustena n. gen. $79: 458$ pretiosa $79: 458$

Eustetha n. gen. 12:296

flaviventris 12:296

glorisa 12:296

limbata 79:462

Galeruca

indica 75:381

submetallescens 79:451

tarsalis 79:452

vittatipennis $75: 380$

Galerucella

annulicornis 46:177

distincta 46:178

placida 75:381

thoracica $86: 33$

tibialis 46:176

tropica $80: 110$

vittaticollis $46: 178$

wallacei $86: 34$

Glytolus n. gen. 90:268

Haplosonyx

concinnus $80: 113$

mouhoti 80:114

quadriplagiata $86: 39$

scutellatus 79:452

sexplagiatus $80: 115$

speciosus $80: 113$

Hoplosoma n. gen.

ornata $86: 27$

ventralis $86: 27$

Hylaspes n. gen. 29:436

assamensis 79:460

longicornis 29:436

Hyphaenia n. gen. $36: 410$

Iphidea n. gen. $31: 127$

discrepens 31:127

Laetana n. gen. 21:232

histrio 21:232

Laphris n. gen. 21:231

emarginata 21:231

Leptarthra n. gen. 12:202

abdominalis $12: 203$

collaris 75:382

dohrnii 12:203

fraternalis 79:455 
Leptoxena n. gen. 93:186 eximea 93:186

Luperodes bella $86: 30$ erythrocephala $75: 380$ jacobyi 86:30 limbella 86:30 marginata 86:30 [homonym, replaced by cincta (WEISE 1921)] pallidulus 46:187

Luperus moorii $46: 188$

Macrima n. gen. $75: 377$ armata 75:377 gracilis 75:378

Malacosoma flaviventre 75:379

Megalognatha n. gen. 76:416 bohemani 76:418 clavicollis $76: 416$ elegans $76: 416$ rufiventre $76: 420$ subcylindrica $76: 419$ suturalis $76: 417$ ventricosa $76: 419$

Melospila n. gen. 12:197 consociata 46:185 nigromaculata 12:297

Menippus metallicus $86: 37$

Merista interrupta 75:382

Mesodonta n. gen. 31:99 marginata 80:111

Metacycla n. gen. 12:206 sallei 12:206

Metalepta n. gen. 12:205 degandii 12:205 fuberculata 12:205

Mimastra n. gen. 34:253 apicalis $86: 28$ arcuata $34: 253$ chennelli 79:450 costata 76:415 limbata 79:449 quadripartita 79:448 soreli $76: 414$

Momaea n. gen. 34:252 viridipennis $34: 252$

Monocesta approximata $82: 258$ sublimbata 82:258

Monolepta covipennis 79:459 cumingii 93:165 fovecollis 93:165 verticalis 93:167 [homonym, replaced by molquensis (ALLARD 1888)] wallacei 93:166

Morphosphaera n. gen. 12:298 maculicollis $12: 298$

Nacrea n. gen. 86:29 apicipennis $86: 29$ maculota $86: 29$

Nadrana n. gen. $34: 250$ pallidicornis 34:251

Nicea n. gen. 27:36 [homonym, replaced by Notonicea (HINCKS 1949)] pulchella 86:35

Ochralea fulva $86: 39$

Oedicerus apicipennis $80: 110$

Oenidea n. gen. 93:159 caeruleipennis 93:159 jacobyi $93: 160$ pallipes 93:161

Oides

antennalis 83:52 costata 83:51 indica 79:443 inornata 79:444

Palpoxena n. gen. 12:203 laeta 12:204

Parastetha n. gen. 79:461 nigricornis 79:461

Paraulaca n. gen. 93:168

Paridea n. gen. 87:26 thoracica $87: 27$

Platyxantha n. gen. 21:233 apicalis 21:234 clypeata 93:158 
nigricornis 21:234

ventralis 21:235

Prasyptera n. gen. 76:411

approximata $76: 413$

distincta $76: 412$

haroldi $76: 414$

nitidipennis $86: 31$

ornata $76: 413$

wallacei $76: 412$

Psetudocophora

brunnea 87:26

distincta 93:171

erichsoni $93: 171$

flaveola $93: 173$

perplexa $93: 175$

wallacei $93: 173$

Sarda n. gen. 31:101 [homonym, repla-

ced by Callisarda (HINCKs 1949)] tetraspilota 31:101

Sastra n, gen. 34:253

discoidalis $86: 35$

limbata 34:254

placida $34: 254$

Stenoplatys n. gen. 12:299

Stethida n. gen. 99:13

Strumatea n. gen. 86:34 nodosa $86: 34$

Syphaxia n. gen. 37:471

Theopea n. gen. 21:237

elegantula 21:238

mouhoti 21:238

pulchella 21:237

Trichidea n. gen. 99:13

bowringii $99: 13$

mouhoti 99:14

Xenarthra n. gen. 12:298

cervicornis 12:299

Xenoda n. gen. 65:225

spinicornis $65: 225$

Hispinae

Acanthodes n. gen. 20:262

favipes 86:118

generosa 20:262

hebe $20: 263$

lateralis 20:265 nigripennis 20:264

tarsata 20:264

Acentroptera n. gen. 4:121

tessellata $4: 122$

Alurnus

batesii $20: 334$

ornatus $39: 87$

salvini $86: 5$

saundersii 4:28

Amblispa n. gen. 4:10

dohrnii 4:12

Anisodera nec 4:101 not Chevrolat

bowringii $4: 102$

excavata 4:105

feae $94: 658$

fraterna 94:655

gestroi $94: 656$

guerinit 4:168

propinqua $94: 657$

scutellata 4:102

sheppardi 4:103

whitei $4: 104$

Arescus

histrio 4:82

perplexus 4:84

separatus $4: 81$

Aspidispa n. gen. 40:378

tibialis $40: 378$

Botryonopa

cyanipennis 4:94

cyanoptera 40:375

imperialis 40:375

sheppardi $4: 92$

spectabilis 4:93

Brachycoryna

fulvipes 86:90

Callispa n. gen. $4: 4$

africana 54:127

bowringii $4: 5$

bretinghami 40:365

brevicornis $40: 365$

cumingii 4:5

dimidiatipennis $4: 7$

elegans 54:126

feae 94:654

fleutiatuxi 98:489 
fortunii $4: 6$

insignis 4:4

mouhoti 40:366

natalensis 4:9

nigricornis 4:8

nigripes 4:9

octopunctata $4: 8$

proxima 40:364

tarsata 40:366

vittata $4: 7$

whitei 4:6

Cephalodonta

abbreviata 86:42

ampliata 86:43

anchoralis $4: 146$

antennata $86: 40$

apicalis 4:158

armata $4: 129$

batesii 4:126

callosa 86:33

championi $86: 36$

chevrolatii $4: 148$

cognata 4:154

collaris $4: 140$

conicicollis $4: 128$

deyrollei $4: 153$

elongata 86:38 [homonyn, replaced

by longula $86: 120$ ]

erudita 86:39

ferox $86: 44$

fraterna 86:44

fryella $4: 137$

fulvolimbata 4:144

gemmans 86:32

godmani $86: 34$

gracilenta $86: 37$

gratiosa 4:142

haroldi $69: 44$

javeti 4:160

laeta $4: 141$

lateralis 4:131

maculata 86:35 [homonym, replaced

by balyi (GRIMSHAW 1897)]

obscurovittata $86: 41$

obsoleta 4:135

octopunctata 4:134 pallida $86: 37$

pascoei $4: 139$

parvula $4: 143$

posticata 86:41

pretiosa 4:135

proxima $86: 39$

pulchella 4:147

pulcherrima 4:132

robinsonii $4: 151$

sallei $4: 150$

scherzeri 4:157

sheppardi 4:133

smithii 4:155

spectabilis 4:139

stevensii $4: 148$

subcornuta 4:138

subparallela $86: 42$

tarsata 4:127

westwoodii $4: 143$

whitei $4: 152$

Cephaloleia

aeneipennis 4:59

affinis 4:44

amazona 40:369

antennata $86: 11$

apicalis $4: 41$

apicicornis $40: 372$

approximata $40: 367$

bella $86: 20$

belti $86: 22$

caeruleata 49:75

championi 86:9

chevrolatii 4:61

clarkella 4:49

cognata 40:372

congener 86:12

consanguinea $86: 23$

corallina 4:42

degandei 4:57

depressa 4:60

deyrollei 4:53

dilaticollis $4: 46$

dimidiaticornis 40:370

discoidalis $86: 15$

distincta $86: 10$

dorsalis 86:15 
elegantula $86: 17$

emarginata 49:74

erichsonii $4: 43$

eximia 4:53

flavipennis 40:373

flavovittata $4: 52$

fryella $4: 62$

fulvipes 4:49

fulvolimbata $86: 24$

funesta $4: 59$

gracilis $69: 41$

gratiosa $4: 40$

grayei 4:51

histrionica $86: 15$

instabilis 86:18

intermedia $86: 19$

lata 86:13

lateralis $86: 17$

laticollis $40: 368$

leptomorpha $86: 25$

leucoxantha $86: 20$

maculipennis 4:58

metallescens $86: 25$

nigriceps 40:370

nigropicta 86:10

opaca $4: 62$

ornata $86: 9$ [homonym, replaced by

ornatrix (DONCKIER 1899)]

perplexa $86: 13$

picta 4:54

placida $86: 11$

pretiosa 4:50

princeps $4: 45$

proxima $4: 47$

pulchella $4: 56$

puncticollis 86:12

quadrilineata $86: 21$

ruficollis 4:165

sallei $4: 45$

saundersii 4:57

semivittata 86:16

separata $86: 22$

stenosoma 86:19

stevensi $86: 26$

suaveola 86:23

subdepressa 69:41 sulciceps $86: 26$

suturalis $86: 14$

tarsata 4:60

tenella 86:26

trimaculata $4: 166$

trivittata $86: 16$

truncatipennis 40:371

vicina 4:55

waterhousei $4: 48$

whitei 4:41

Chaeridiona $\mathrm{n}$. gen. 40:380

metallica $40: 381$

picea $40: 382$

Chalepus

alienus 86:78

amabilis $86: 65$

amiculus 86:74

amicus 86:54

amplipennis $86: 82$

angulosus $86: 73$

breviceps $86: 57$

bivittaticollis $86: 121$

championi 86:50 [note on 86:120 sta-

tes this species belongs in Uroplata]

chapuisi 86:81

chromaticus 86:70

clypeatus $86: 71$

congener 86:52

consanguineus $86: 67$

contiguus $86: 64$

contubernalis $86: 80$

digressus 86:66

distinctus 86:56

diversipes 86:59

fraternalis $86: 122$

fraternus 86:64

fryi $86: 59$

generosus $86: 76$

haematoderus 86:69

hepburni 86:76

horni $86: 72$

instabilis 86:60

jansoni 86:55

lateralis $86: 75$

marginatus $86: 75$

marmoratus 86:60 
mediolineatus $86: 122$

nigripictus 86:66

opacicollis $86: 53$

palmeri 86:85

pascoei 86:65

pallescens $86: 56$

placidus $86: 52$

posticatus $86: 77$

productus $86: 63$

propinquus 86:71

rufithorax 86:70

sanguinosus $86: 70$

saundersi $86: 53$

semilimbatus 86:51

signaticollis $86: 86$

similatus 86:73

subapicalis $86: 61$

subhumeralis $86: 74$

subparallelus $86: 72$

suspiciosus 86:77

suturalis $86: 51$

terminatus 86:54

tibialis 86:55

univittatus 86:84

vicinalis $86: 67$

viridanus $86: 62$

vittaticollis $86: 62$

waterhousii $86: 81$

Chaeridonota $\mathbf{n}$. gen. 40:380

Charispa n. gen. 49:73

amicula 49:73

caerulescens 49:74

elongata 49:73

Charistena n. gen. 22:251

basalis 22:253

bellula 22:253

championi 86:46

deyrollei $22: 253$

elegantula 22:254

funesta $86: 46$

lecontii 22:252

pilatei 22:254

trilineata 22:255

Cheirispa n. gen. 4:71

bidentata $4: 72$

distincta $86: 30$ dorsata $86: 29$

suturalis 4:72

Cladispa n. gen. 4:22

quadrimaculata 4:23

Coelaenomenodera tarsata 4:119

Cryptonychus

bipunctatus $4: 78$

dubius 4:77

murrayi 4:76

Demotispa n. gen. $4: 65$

bimaculata $4: 68$

elegans 49:75

grayella 4:66

limbata 86:27

pallida 4:65

pulchella 4:67

sallei $4: 167$

tibialis $4: 66$

Distolaca n. gen. 4:118

apicalis 4:118

whitei $4: 117$

Downesia n. gen. 4:107

atrata $40: 377$

basalis $94: 662$

fulvipennis 94:660

gestroi 94:660

insignis 4:107

picea 94:661

strigicollis 54:128

tarsata 40:377

Estigmena

terminalis $40: 376$

Eurispa n. gen. 4:85

howittii 39:90

normalis $39: 89$

odewahnii 39:88

vittata 4:86

Euxema n. gen. 86:3

insignis $86: 3$

Gonophora

apicalis $4: 116$

apicipennis 40:379

bowringii $4: 113$

chalybeata $4: 115$

chapuisi 54:129 
crassipes $69: 43$

fuscipes $4: 111$

horsfieldi $69: 43$

insignis 4:111

integra $4: 114$

lineata $69: 42$

masoni 92:85

nigriceps 40:379

pallida $4: 115$

saundersii $4: 110$

suturella $4: 110$

tibialis 69:42

wallacei 4:109

Hispa

abdominalis 94:664

aenescens $91: 412$

confluens $98: 490$

discoidalis $94: 664$

fleutiauxi 98:491

japonica 46:215

melanosticta $98: 492$

moerens 46:215

perraudierei 98:491

reticulata $94: 665$

subquadrata 46:216

Hispodonta n. gen. 4:78

elegantula $40: 374$

nigricomis 4:79

plagiata 90:270

Hispoleptis n. gen. 20:262

Hispopria n. gen. 4:94

bipunctata 4:96

foveicollis 4:96

geniculata 4:97

grandis 4:95

terminalis 54:127

Homalispa n. gen. $4: 33$

apicalis $4: 34$

armata $4: 34$

batesii $4: 36$

deyrollei 4:36

gracilis $86: 8$

grayella $4: 35$

javeti 4:38

limbifera $86: 7$

marginata 4:33 variabilis $86: 7$

vespertina $4: 37$

Javeta n. gen. 4:108

Leptispa n. gen. 4:1

abdominalis $4: 3$

allardi 98:489

godwini $40: 364$

natalensis $4: 2$

pygmaea 4:2

Macripsa n. gen. 4:90 saundersii $4: 91$

Melanispa n. gen. 4:30 truncata $4: 31$

Metaxycera $n$. gen, 21:255

amazona 22:257

rubroguttata 22:256

sexpustulata 22:258

Microrhopala n. gen. 20:268

bivitticollis $20: 270$

perforata $20: 269$

pulchella 20:270

sallei $20: 271$

Monochirus

moestus 94:662

Octotoma

championi $86: 88$

Oediopalpa n. gen. 4:16

basalis $4: 17$

caerulea 4:21

collaris $4: 19$

cyanipennis $4: 18$

fulvipes $4: 21$

guerinii $4: 20$

laticollis $4: 19$

nigripes $4: 17$

Oxycephala

depressa 4:74

imperialis 5:88

wallacei $90: 450$

Parachalepus n. gen. 86:47 brevicornis 86:48

Prionispa gemmata 54:129

Promecotheca callosa 54:128

cumingii $4: 88$ 
reichii $40: 374$

varipes $4: 88$

Prosopodonta n. gen. 4:68

costata 86:29 [homonym, replaced by

balyi (WEISE 1905)]

limbata 4:69

proxima $4: 69$

rufipennis 4:70

Stenispa n. gen. $4: 13$

attenuata $63: 185$

batesii 4:15

brevicornis $86: 4$

clarkella 4:15

collaris 4:163

parryi 4:14

sallei $4: 164$

vespertina $63: 184$

vicina $4: 15$

Stethispa n. gen. 20:265

bonvouloirii 20:265

confusa 20:267

conicicollis 20:267

gratiosa 20:266

Uroplata

armata 86:110

armigera $86: 114$

beata $86: 99$

bellicosa $86: 99$

belti $86: 106$

buqueti 86:107

championi 86:120 [see note under

Chalepus]

cincta 20:342

clypeata $86: 105$

concava 20:342

consociata 86:101

coxalgica $86: 123$

cruentata 20:340

decepta $86: 104$

dimidiata 86:103

distincta 86:96

distinguenda $86: 111$

dolorosa $86: 116$

duodecimmaculata 24:351

elegantula 86:103

elevata 86:107 emorsitans 86:102

excisa $86: 111$

explanata $86: 93$

fulvescens 86:101

fulvopicta 86:109

fulvopustulata 86:113

grayi 24:354

haematopyga $86: 108$

limbata 86:106

lineaticollis $86: 113$

melancholica 86:116

miniata 20:344

militaris 20:335

nebulosa 86:115

octopustulata 20:341

pascoei 86:109

pectoralis 20:339

pretiosa $20: 338$

puella 20:337

robinsonit $20: 343$

sallaei 86:96

sanguinipennis $86: 123$

stalei 20:339

stevensi $24: 353$

submarginalis 20:337

terminalis 24:355

sexdecimguttata $24: 356$

variegata $86: 114$

vittatipennis $86: 95$

walkeri $24: 352$

westwoodi 86:112

Wallacea n. gen. 4:97 [homonym, repla-

ced by Wallaceana (MAULK 1928)]

bowringii 4:99

collaris 4:98

distinguenda 4:99

Xanthispa n. gen. 4:31

caeruleipennis $86: 6$

Xenispa n. gen. 4:63

pulchella 4:64

Lamprosomatinae

Lamprosoma

armatum 77:351

amazonum 77:348

batesi $77: 347$ 
canaliculatum $77: 349$

cuneatum 77:350

cupreatum 45:82

cupricolle $77: 348$

hypochryseum 77:347

nigrocaeruleum 45:83

tricolor 77:346

tridentatum 77:349

$$
\begin{array}{r}
\text { Megalopinae } \\
\text { Agathomerus } \\
\text { batesii } 6: 199 \\
\text { sallei } 8: 153 \\
\text { Mastostethus }
\end{array}
$$

basalis 55:127

batesii $6: 201$

buckleyi $55: 124$

dohrnii 12:281

fraternus 55:128

jansoni 55:126

javeti 6:203

jekelii $6: 202$

lavatus 12:281

pascoei $6: 202$

philemon 17:616

picticollis $55: 123$

pictus 55:125

placidus 55:127

speciosus 55:124

stalii 12:282

thoracicus 6:200

Megalopus

elongatus 55:128

vittaticollis 55:129

waterhousei 6:203

Pedrilla

annulata 45:79

Poecilomorpha

amabilis 61:177

fulvipennis 6:209

murrayi 6:208

thoreyi 25:436

Temnaspis

downesii 6:205

insignis 6:208

japonicus $45: 78$ mowhoti $25: 435$

nigriceps 6:207

pulcher 6:206

quinquemaculatus 6:206

speciosus 6:204

westwoodii 28:41

Megascelis

basalis $77: 340$

elegans 12:276

femorata 77:339

posticata 77:339

Orsodacna

Orsodacninae

variabilis $68: 337$

Sagrinae

Carpophagus

excavatus 41:382

Duboulaia n. gen. 41:381

flavipennis 41:382

fulva 50:45

rugosa 50:46

Polyoptilus

pachytoides $50: 47$

pascoei $50: 47$

waterhousii $50: 46$

Sagra

dohrnii 10:253

emarginata 10:246

ferox $77: 338$

jansoni 10:243

javeti 10:240

kirbyi 10:250

livingstonii $25: 434$

longipes $77: 337$

lucida $10: 245$

mouhoti 12;193

murrayi 10:251

mutabilis 25:433

parryi 10:247

pfeifferi 10:239

stevensi 10:241 
Synetinae

Syneta adamsi $68: 378$

\author{
Zeugophorinae \\ Zetigophora \\ kirbyi $32: 183$
}

\section{Literature Cited}

AlL,ARD, E. 1888: Diagnoses de Coléoptères nouveaux du genre Monolepta. -LeNaturaliste (2)10: 260270.

ChapUs, F. 1874: In J. T. LACORDARE, Histoire naturelle des insectes. Genera des coléoptères. Volume 10, Famille des Phytophages. Paris. $-455 \mathrm{pp}$.

Clavareau, H. 1904: In M. Jacoby \& H. Clavareau, Coleoptera Phytophaga, Fam. Crioceridae. In P. WrtsmaN (ed.). Genera Insectorum, fasc. 23. - Brussels.

CSIKI, E. 1939: In F. HEIKERTINGER \& E. CSIKa. Coleopterorum Catalogus. Chrysomelidae: Halticinae I. - pars 166: 1-336.

CSIKI, E. \& HeIKERTINGER, F. 1940: In F. HeIKERTINGER \& E. CSIKI, Coleopterorum Catalogus. Chrysomelidae: Halticinae II. - pars 166: 337-635.

DonCKIER, H. H. 1899: Catalogue systematique des Hispides. - Annales de la Société Entomologique de France 68: 540-615.

DUVIVIER, A. 1885: Catalogue des chrysomélides, haltịcides et galérucides décrites postérieurement a la publication du catalogue de Munich. - Mémoires de la Société Royal des Sciences de Liège (2)I1: $1-64$.

GAHAN, C. J. 1891: Notes on some species of Diabrotica. - Proceedings of the Entomological Society of London 1890: xliii.

GEMMINGER, M. \& HAROLD, E. von 1874: Catalogus coleopterorum hucusque descriptorum synonymicus et systematicus. - Volume 11. Chrysomelidae. pars 1.

GrIMSHAw, P. H. 1897: On some type specimens of Lepidoptera and Coleoptera in the Edinburgh Museum of Science and Art. - Transactions of the Royal Society of Edinburgh 39: 1-11.

HaROLD, E. von 1874: Geănderte Namen. - Coleopterologische Hefte 12: 152.

HAROLD, E. von 1875: Geänderte Namen, - Coleopterologische Hefte 13: 185.

HincKs, W. D. 1949: Some nomenclatorial notes on Chrysomelidae (Col.). No. 1, Galerucinae. - The Annals and Magazine of Natural History (12)2: 607-622.

JACOBSON, G. G. 1922: Chrysomelidae palaearctici novi vel parum cogniti (Coleoptera) IV. - Annuaire da Musée Zoologique de l'Académie des Sciences de Russie, Petrograd 25: 517.534.

JACOBY, M. 1884: Galerucinae. In F. D. GODMAN \& O. SALVIN (eds.). - Biologia Centrali-Americana. Zoology, Insecta, Coleoptera, Phytophaga. 6(1): 265-336.

$\mathrm{J}_{\text {ACOBY }}$ M. 1887: Galerucidae. In F. D. GODMAN \& O. SALVIN (eds.). - Biologia Centrali-Americana. Zoology, Insecta, Coleoptera, Phytophaga. 6(1): 497-584.

JACOBY, M. 1908: The fauna of British India including Ceylon and Burma. Coleoptera. Chrysomelidae. Volume 2. - London. - $534 \mathrm{pp}$.

KMOTO, S. 1985: Check-list of Chrysomelidae of South East Asia, south of Thailand and west of IrianJaya of Indonesia III. Eumolpinae, 1. - Kurume University Journal 34: 57-87.

LEFEVRE, E. 1885: Eumolpidarum hucusque cognitarum catalogus, sectionum conspectu systematico, generum sicut et specierum nonnullarım novarum descriptionibus adjunctis. - Mémoires de la Société Royale des Sciences de Liège (2)11(16): 1-172.

MAULIK, S. 1928: New chrysomelid beetles from India with a note on the scales of Coleoptera. Proceedings of the Zoological Society of London 1928: 151-161.

SEENO, T. N. \& WILCOX, J. A. 1982: Leaf beetle genera (Coleoptera: Chrysomelidae). - Entomography 1: 1-221.

Uhmann, E. 1957: Coleopterorum Catalogus Supplementa. Chrysomelidae: Hispinae, Hispinae Americanae, - pars 35(1): 1-153. 
UhMANN, E. 1958: Coleopterorum Catalogus Supplementa. Chrysomelidae: Hispinae, Hispinae Africanae, Eurasiaticae, Australicae - pars 35(2): 155-398.

WEISE, J. 1905: Beschreibung einiger Hispinen. - Archiv für Naturgeschichte 71: 49-104.

WEISE, J. 1916: Synonymische Mitteilungen. - Deutsche Entomologische Zeitschrift 1916: $37-41$.

WEISE, J. 1921: Wissenchaftliche Ergebrisse du schwedischen entomologischen Reise des Herm A. ROMAN in Amazonas 1914-1915. 6. Chrysomelidae. - Arkiv för Zoologi 14: 1-205.

WucoX, J. A. 1971: Coleopteronum Catalogus Supplementa. Chrysomelidae: Galerucinae, Oidini, Galerucini, Metacyclini, Sermylini. - pars 78(1): 1-220.

WILcox, J. A. 1972: Coleopterorum Catalogus Supplementa. Chrysomelidae: Galerucinae, Luperini: Aulacophorina, Diabroticina. - pars 78(2): 221-431.

Wucox, J. A. 1972: Coleopterorum Catalogus Supplementa. Chrysomelidae: Galerucinae, Luperini: Luperina. - pars 78(3): 433-664.

\author{
Author's addresses: \\ Charles L. STANES \& Sus an L. STANES \\ 3302 Decker Place \\ Edgewater, Maryland 21037 \\ U.S.A.
}

\title{
Besprechungen
}

SAbELLA, G.: Pselafidi di Sicilia. - Torino: Museo Regionale di Scienze Naturali, 1998. - 415 S.: 190 fig., 38 Fot. - (Monografie; XXV). - L 140.000

Die Pselaphidae (inzwischen Staphylinidae: Pselaphinae) sind in Sizilien durch 82 Arten und Unterarten vertreten, die zu 30 Gattungen gehören. Von diesen Arten und Unterarten sind 27 (33\%) endemisch. Diese lokalen Endemiten machen den besonderen Wert der Pselaphinen-Fauna Siziliens aus. Die Monographie der Pselaphinae Siziliens basiert auf dem Material der Museumssammlungen und auf etwa 9.000 Exemplaren, die vorm Autor in 15 Jahren Feldforschung selbst gesammelt worden sind. Zehn der 27 endemischen Arten und Unterarten sind durch den Autor, teilweise als Koautor, beschrieben worden. Er hat mehr als jeder andere zur Erforschung der Pselaphinae Siziliens beigetragen. Die vorliegende Monographie faßt das gesamte Wissen fur Sizilien sehr übersichtlich zusarnmen. Sie enthält Bestimmungstabellen für alle Triben, Gattungen und Arten. Für jede Art gibt es eine komplette Bibliographie, eine Liste der Synonyme, Angaben zur geographischen Verbreitung, eine Verbreitungskarte auf UTM-Basis, kurze okologische Angaben und die Angabe der jeweiligen chorologischen Kategorie. In einem Kapitel werden die Zusammensetzung und die Biogeographie der Pselaphinen-Fauna Siziliens diskutiert. Das Buch ist reich illustriert. Es enthält nicht nur Seiten füllende Habitus- und Genital-Zeichnungen, sondem auch 38 Fotos von Biotopen und Landschaften auf Sizilien, die beim Rezensenten die Sehnsucht wecken, dort einmal selbst zu sammein.

L. ZERCHE 